\title{
$R$-EQUIVALENCE IN ADJOINT CLASSICAL GROUPS OVER FIELDS OF VIRTUAL COHOMOLOGICAL DIMENSION 2
}

\author{
AMIT KULSHRESTHA AND R. PARIMALA
}

Dedicated to our teacher Professor R. Sridharan on his seventieth birthday.

\begin{abstract}
Let $F$ be a field of characteristic not 2 whose virtual cohomological dimension is at most 2. Let $G$ be a semisimple group of adjoint type defined over $F$. Let $R G(F)$ denote the normal subgroup of $G(F)$ consisting of elements $R$-equivalent to identity. We show that if $G$ is of classical type not containing a factor of type $D_{n}, G(F) / R G(F)=0$. If $G$ is a simple classical adjoint group of type $D_{n}$, we show that if $F$ and its multi-quadratic extensions satisfy strong approximation property, then $G(F) / R G(F)=0$. This leads to a new proof of the $R$-triviality of $F$-rational points of adjoint classical groups defined over number fields.
\end{abstract}

\section{INTRODUCTION}

In [Ma, Chapter II, $\S 14]$ Manin introduced the notion of $R$-equivalence on a variety $X$ over a field $F$ as follows : two points $x, y \in X(F)$ are $R$-equivalent if there exist $x=x_{0}, x_{1}, x_{2}, \cdots, x_{n}=y \in X(F)$ and $F$-rational maps $f_{i}: \mathbb{P}^{1} \rightarrow X, 1 \leq i \leq n$, regular at 0 and 1 such that $f_{i}(0)=x_{i-1}$ and $f_{i}(1)=x_{i}$. If $X$ is the underlying variety of a connected algebraic group $G$, then the set of elements of $G(F)$ which are $R$-equivalent to 1 is a normal subgroup $R G(F)$ of $G(F)$. We denote the quotient $G(F) / R G(F)$ by $G(F) / R$. A connected algebraic group is called $R$-trivial, if for all field extensions $E$ of $F$, we have $G(E) / R=0$. Colliot-Thélène and Sansuc [CTS] proved that if the variety of a connected algebraic group $G$ is stably rational, then $G$ is $R$-trivial. For example, if $G$ is an adjoint classical group of type ${ }^{1} A_{n},{ }^{2} A_{2 n}$ [VK, pp. 240] or $B_{n}$, then $G$ is rational, and hence $R$-trivial.

Let $G$ be a classical group of adjoint type defined over a number field and $\tilde{G}$ be a simply connected cover of $G$.

(i) $[\mathrm{Y}, \mathrm{CM}]$ If $\tilde{G}$ is of type ${ }^{2} A_{n}$, then $\tilde{G}(F) / R=0$.

(ii) [PR, Theorem 9.5] The group $\tilde{G}(F)$ is projectively simple provided $\tilde{G}$ does not contain a factor of type $A_{n}$. In particular the non-central normal subgroup $R \tilde{G}(F)$ coincides with $\tilde{G}(F)$.

Further by [G, Corollaire III.4.2], the natural map $\tilde{G}(F) / R \rightarrow G(F) / R$ is surjective. In view of this together with (i) and (ii) above, we deduce that $G(F) / R=0$

Received by the editors July 31, 2005.

2000 Mathematics Subject Classification. Primary 20G15, 14G05.

Key words and phrases. Adjoint classical groups, $R$-equivalence, algebras with involutions, similitudes. 
for classical groups $G$ of adjoint type over number fields. The proof of Gille for the surjectivity of $\tilde{G}(F) / R \rightarrow G(F) / R$ for number fields uses besides his results on the norm principle, deep arithmetic results due to Kato-Saito [KS, Theorem 4] and Sansuc [S, Corollaire 3.5.c], which do not admit analogues over general fields of virtual cohomological dimension two. In fact, simply connected groups $\tilde{G}$ of type $C_{n}$ are rational, and a surjectivity statement $\tilde{G}(F) / R \rightarrow G(F) / R$ would immediately lead to the triviality of $G(F) / R$ for adjoint groups $G$ of type $C_{n}$.

Number fields are examples of fields of virtual cohomological dimension two. The aim of this paper is to study the group $G(F) / R$ where $G$ is a classical group of adjoint type defined over a field of virtual cohomological dimension two.

Let $\Gamma_{F}$ be the Galois group $\operatorname{Gal}\left(F_{s} / F\right)$, where $F_{s}$ is the separable closure of $F$. The cohomological dimension of $F$ is the least positive integer $n$ such that for all discrete torsion $\Gamma_{F}$-modules $M$, the Galois cohomology groups $H^{i}\left(\Gamma_{F}, M\right)$ are zero for all $i \geq n+1$. A field $F$ is said to have virtual cohomological dimension $n$ if the cohomological dimension of $F(\sqrt{-1})$ is $n$. We write $\operatorname{cd}(F)$ to denote the cohomological dimension and $\operatorname{vcd}(F)$ to denote the virtual cohomological dimension of $F$. We prove that $G(F) / R=0$ for adjoint groups $G$ of type ${ }^{2} A_{n}$ and $C_{n}$ over a field $F$ of virtual cohomological dimension at most 2. For classical groups of type $D_{n}$, we prove that if the cohomological dimension of $F$ is at most 2, then $G(F) / R=0$. Further, if the virtual cohomological dimension of $F$ is at most 2, then we show that $G(F) / R=0$, provided $F$ satisfies certain approximation properties. These results, in particular, lead to a new proof of the triviality of $G(F) / R$ for adjoint classical groups over number fields.

The main ingredients in proofs of our results are Merkurjev's computation of $G(F) / R$ for all adjoint groups of classical type [Me2, Th. 1], as well as results on the classification of hermitian forms over division algebras with involution over fields of virtual cohomological dimension two [BP2].

\section{SOME KNOWn RESUlts}

In this section, we record some known results which are used in the paper. Let $F$ be a field with $\operatorname{char}(F) \neq 2$. Let $Z=F$, or a quadratic extension of $F$. Let $A$ be a central simple algebra over $Z$ and $\sigma$ be an involution on $A$ of either kind. If $\sigma$ is of the second kind, let $Z^{\sigma}=F$. An element $a \in A^{*}$ is said to be a similitude of $(A, \sigma)$ if $\sigma(a) a \in F^{*}$. The similitudes of $(A, \sigma)$ form a group which we denote by $\operatorname{Sim}(A, \sigma)$. The map $\mu(a)=\sigma(a) a$ is a homomorphism $\mu: \operatorname{Sim}(A, \sigma) \rightarrow F^{*}$ whose image is denoted by $G(A, \sigma)$. Elements of $G(A, \sigma)$ are called multipliers. Let $\sigma$ be adjoint to a hermitian form $h$. Then $\lambda \in G(A, \sigma)$ if and only if $\lambda h \simeq h$ [KMRT, Prop. 12.20]. Let $\operatorname{Sim}(A, \sigma)$ denote the algebraic group whose $F$ rational points are given by $\operatorname{Sim}(A, \sigma)$. Let $\operatorname{Sim}_{+}(A, \sigma)$ be the connected component of identity of $\operatorname{Sim}(A, \sigma)$. Let $\operatorname{Sim}_{+}(A, \sigma)$ denote the $F$-rational points of $\operatorname{Sim}_{+}(A, \sigma)$. Elements of $\operatorname{Sim}_{+}(A, \sigma)$ are called proper similitudes. We denote the group $\mu\left(\operatorname{Sim}_{+}(A, \sigma)\right)$ by $G_{+}(A, \sigma)$. Let $R_{Z / F}$ denote the Weil restriction to $F$. The group of projective similitudes is the quotient group

$$
\operatorname{Sim}(A, \sigma) / R_{Z / F}\left(\mathbf{G}_{m}\right)
$$

which we denote by $\operatorname{PSim}(A, \sigma)$. The group of $F$-rational points of $\operatorname{PSim}(A, \sigma)$ is $\operatorname{Sim}(A, \sigma) / Z^{*}$. The connected component of the identity of the $\operatorname{group} \operatorname{PSim}(A, \sigma)$ is denoted by $\operatorname{PSim}_{+}(A, \sigma)$ 
Let $N(Z)=F^{* 2}$ or $N_{Z / F}\left(Z^{*}\right)$ according to whether $\sigma$ is of the first kind or second kind, respectively. Let $\operatorname{Hyp}(A, \sigma)$ be the subgroup of $F^{*}$ generated by the norms from all those finite extensions of $F$, where the involution $\sigma$ becomes hyperbolic. If $A$ is split, the involution $\sigma$ is adjoint to a quadratic form $q$ over $F$. The group $G_{+}(A, \sigma)$ is then denoted by $G_{+}(q)$, and the group $G(A, \sigma)$ is denoted by $G(q)$. In fact $G_{+}(q)=G(q)$, because of the existence of hyperplane reflections in the orthogonal group.

Theorem 1.1 ([Me2, Th. 1]). With notation as above, $N(Z) . \operatorname{Hyp}(A, \sigma)$ is a subgroup of $G_{+}(A, \sigma)$ and further,

$$
\operatorname{PSim}_{+}(A, \sigma)(F) / R \simeq G_{+}(A, \sigma) / N(Z) . \operatorname{Hyp}(A, \sigma) .
$$

We now record a lemma due to Dieudonné.

Lemma 1.2 (Dieudonné, [KMRT, Lemma 13.22]). Let $q$ be a quadratic form of even rank and $d=\operatorname{disc}(q)$. Let $L=F(\sqrt{d})$. Then $G(q) \subseteq N_{L / F}\left(L^{*}\right)$.

The following result of Merkurjev-Tignol extends Dieudonné's lemma.

Lemma 1.3 ([MT, Th. A]). Let $A$ be a central simple algebra of even degree with an orthogonal involution $\sigma$. Let $d=\operatorname{disc}(\sigma)$ and let $L=F(\sqrt{d})$. Then $G_{+}(A, \sigma) \subseteq N_{L / F}\left(L^{*}\right)$.

Let $q$ be a non-degenerate quadratic form of rank $r$ over $F$. Let $\tau_{q}$ be the adjoint involution on $M_{r}(F)$. Then $\operatorname{Hyp}\left(M_{r}(F), \tau_{q}\right)=\operatorname{Hyp}(q)$, the subgroup of $F^{*}$ generated by $N_{L / F}\left(L^{*}\right)$, with $L$ varying over finite extensions of $F$ where $q$ becomes hyperbolic. If $r$ is odd, then $\operatorname{Hyp}(q)=1$.

Theorem 1.4 ([Me2, pp. 200]). Let $A$ be a central simple algebra of odd degree with an orthogonal involution $\sigma$. Let $q$ be a quadratic form over $F$ such that $\sigma$ is adjoint to $q$. Then $G_{+}(A, \sigma)=G(q)=\operatorname{Hyp}(q) \cdot F^{* 2}=\operatorname{Hyp}(A, \sigma) \cdot F^{* 2}=F^{* 2}$.

We now record a result due to Knebusch which describes the group of spinor norms of a quadratic form. Let $q$ be a quadratic form over $F$ and $\operatorname{sn}(q)$ denote the subgroup of $F^{*}$ generated by $F^{* 2}$ and representatives of the square classes in the image of the spinor norm map sn : $\mathrm{SO}(q) \rightarrow F^{*} / F^{* 2}$. For a central simple algebra $A$ over $F$, let Nrd $: A \rightarrow F$ denote the reduced norm map. For $S \subseteq F^{*}$, we denote by $\langle S\rangle$, the subgroup generated by $S$ in $F^{*}$.

Theorem 1.5 (Knebusch's norm principle, [L, Theorem VII.5.1]). For a quadratic form $q$ over $F$ we have:

$\operatorname{sn}(q)=\left\langle\left\{N_{L / F}\left(L^{*}\right): L / F\right.\right.$ is a quadratic extension over $F$ and $q_{L}$ is isotropic $\left.\}\right\rangle$.

The two results recorded below describe the group $G(A, \sigma)$ in the case when $\sigma$ is unitary or symplectic under further assumptions on the degree of $A$.

Theorem 1.6 ([Me2, §2]). Let $F$ be a field with $\operatorname{char}(F) \neq 2$. Let $A$ be a central simple algebra over $Z$ of odd degree with an involution $\sigma$ of second kind with $Z^{\sigma}=F$. Then $G_{+}(A, \sigma)=G(A, \sigma)=\operatorname{Hyp}(A, \sigma)=N(Z)$.

Theorem 1.7 ([Me2, §2, Lemma 3]). Let $F$ be a field with $\operatorname{char}(F) \neq 2$. Let $A$ be a central simple algebra over $F$ of degree $2 n$ with $n$ odd. Let $\sigma$ be a symplectic involution on $A$. Then $G_{+}(A, \sigma)=G(A, \sigma)=\operatorname{Hyp}(A, \sigma)=\operatorname{Nrd}(A)$. 
The next results we record are local criteria for elements to be reduced norms or spinor norms for formally real fields $F$ with $\operatorname{vcd}(F) \leq 2$.

Theorem 1.8 ([BP2, Theorem 2.1]). Let $F$ be a formally real field with $\operatorname{vcd}(F) \leq 2$. Let $\Omega$ denote the set of orderings on $F$. Let $A$ be a central simple algebra over $F$ and $A_{v}=A \otimes_{F} F_{v}, F_{v}$ denoting the real closure of $F$ at $v$. Let $\lambda \in F^{*}$ be such that $\lambda>_{v} 0$ at those orderings $v \in \Omega$ where $A_{v}$ is non-split. Then $\lambda \in \operatorname{Nrd}\left(A^{*}\right)$.

Theorem 1.9 ([BP2, Cor. 7.10]). Let $F$ be a formally real field with $\operatorname{vcd}(F) \leq 2$. Let $q$ be a quadratic form over $F$. Then sn $(q)$ consists of elements of $F^{*}$ which are positive at each $v \in \Omega$ such that $q$ is definite at $F_{v}$.

We say that a quadratic form $q$ over $F$ is locally isotropic if over each real closure $F_{v}, v \in \Omega$, the form $q$ is isotropic.

Corollary 1.10. With notation as in 1.9, if is $q$ locally isotropic, then $\operatorname{sn}(q)=F^{*}$.

Let $\Gamma_{F}$ denote the Galois group $\operatorname{Gal}\left(F_{s} / F\right)$. For a discrete $\Gamma_{F}$-module $M$, let $H^{n}(F, M)$ denote the Galois cohomology group $H^{n}\left(\operatorname{Gal}\left(F_{s} / F\right), M\right)$. We now record some results of Arason which we shall use in the paper.

Theorem 1.11 (Corollary 4.6, [A1]). Let $Z=F(\sqrt{\delta})$ be a quadratic extension of $F$. Then we have a long exact sequence of abelian groups

$$
\cdots \rightarrow H^{n}\left(F, \mu_{2}\right) \stackrel{\text { res }}{\longrightarrow} H^{n}\left(Z, \mu_{2}\right) \stackrel{\text { cores }}{\longrightarrow} H^{n}\left(F, \mu_{2}\right) \stackrel{\cup_{n, 1}(\delta)}{\longrightarrow} H^{n+1}\left(F, \mu_{2}\right) \rightarrow \cdots
$$

where res and cores denote the restriction and corestriction maps respectively.

In view of 1.11 and the isomorphism $H^{2}\left(F, \mu_{2}\right) \simeq{ }_{2} \operatorname{Br}(F)$, we have the following.

Proposition 1.12. Let $Z=F(\sqrt{\delta})$ be a quadratic extension of $F$ and let $A$ be a central simple algebra over $Z$ with $\exp (A)=2$ and $\operatorname{cores}_{Z / F}([A])=0 \in H^{2}\left(F, \mu_{2}\right)$. Then there exists a central simple algebra $A_{0}$ over $F$ such that $A_{0} \otimes_{F} Z$ is Brauer equivalent to $A$.

We say that a field extension $L / F$ is a quadratic tower over $F$ if there exist fields $F_{i}$ such that $F=F_{0} \subseteq F_{1} \subseteq \cdots \subseteq F_{r}=L$ and each $F_{i} / F_{i-1}$ is a quadratic extension for $1 \leq i \leq r$. We denote by $\mathcal{F}_{2}(F)$ the set of quadratic towers of $F$ in an algebraic closure of $F$. Let $I(F)$ denote the fundamental ideal of the Witt ring $W(F)$ of $F$. For each $n \geq 1$, we denote by $I^{n}(F)$, the ideal $I(F)^{n}$.

Lemma 1.13 ([A1, Satz 3.6]). Let $I^{3}(F)=0$ and $L / F$ be a quadratic tower. Then $I^{3}(L)=0$.

Theorem 1.14 ([A2, Prop. 2]). Let $F$ be a field with $\operatorname{cd}(F) \leq 2$. Then $I^{3}(F)=0$.

A non-trivial element $\chi \in H^{r}\left(F, \mu_{2}\right)$ is called $(-1)$-torsion-free if for every $s \geq 1$, the element $\chi \cup(-1) \cup(-1) \cup \cdots \cup(-1) \in H^{r+s}\left(F, \mu_{2}\right)$ is non-trivial. The following is a consequence of 1.11

Proposition 1.15. Let $F$ be a field with $\operatorname{vcd}(F) \leq n$. Then $H^{n+1}\left(F, \mu_{2}\right)$ is $(-1)$ torsion-free.

The following lemma relates the conditions $\operatorname{vcd}(F) \leq 2$ and $I^{3}(F)$ being torsionfree.

Lemma 1.16 ([BP2, Lemma 2.4]). Let $F$ be a field with virtual cohomological dimension at most two. Then $I^{3}(F)$ is torsion-free. 
Proof. Since $\operatorname{vcd}(F) \leq 2$, by $[\mathrm{AEJ}]$ the invariants $e_{r}: I^{r}(F) \rightarrow H^{r}\left(F, \mu_{2}\right)$ have kernel $I^{r+1}(F)$ for each $r \geq 0$ and $H^{r}\left(F(\sqrt{-1}), \mu_{2}\right)=0$ for $r \geq 3$. Then it is evident from Arason exact sequence 1.11 for the quadratic extension $F(\sqrt{-1}) / F$ that $H^{r}\left(F, \mu_{2}\right) \stackrel{\cup(-1)}{\longrightarrow} H^{r+1}\left(F, \mu_{2}\right)$ is an isomorphism for $r \geq 3$. Let $q \in I^{3}(F)$ be a torsion-element. Then $2^{s} \cdot q=0 \in W(F)$ for some integer $s \geq 0$. As a consequence $e_{3}(q) \cup(-1) \cup(-1) \cup \cdots \cup(-1)=0 \in H^{3+s}\left(F, \mu_{2}\right)$. Since $H^{r}\left(F, \mu_{2}\right) \stackrel{\cup(-1)}{\longrightarrow}$ $H^{r+1}\left(F, \mu_{2}\right), r \geq 3$, are isomorphisms, we conclude that $e_{3}(q)=0$; i.e. $q \in$ $\operatorname{ker}\left(e_{3}\right)=I^{4}(F)$. By a similar argument $q \in I^{r}(F)$ for each $r \geq 3$ and hence $q \in \bigcap_{r} I^{r}(F)$. By a theorem of Arason-Pfister [L, Cor. X.3.2], $q=0 \in W(F)$ and hence $I^{3}(F)$ is torsion-free.

The following result is a weaker form of [Se, Prop. 10, §II.4.1].

Theorem 1.17. Let $F$ be a field and $\operatorname{cd}(F) \neq \operatorname{vcd}(F)$. Then $F$ has orderings.

\section{SOME NORM PRINCIPLES}

Let $F$ be a field with $\operatorname{char}(F) \neq 2$ and $I^{3}(F)=0$. Let $A$ be a central simple algebra with $\exp (A)=2$. Then by [Me1], there are quaternion algebras $H_{i}, 1 \leq$ $i \leq r$, such that $A \sim H_{1} \otimes H_{2} \otimes \cdots \otimes H_{r}$. We define an integer $r(A)$ associated to $A$ as follows:

$$
r(A):=\min \left\{r: A \sim H_{1} \otimes H_{2} \otimes \cdots \otimes H_{r}\right\} .
$$

If $A$ is split, then we define $r(A)=0$. Given a central simple algebra $B$ over a field $Z$ with $[Z: F] \leq 2$ and a field extension $L$ of $F$, we set $B_{L}=B \otimes_{F} L$.

Proposition 2.1. Let $I^{3}(F)=0$ and $A$ be a central simple algebra over $F$. If $\exp (A)$ is a power of 2 , then

$$
F^{*}=\left\langle\left\{N_{L / F}\left(L^{*}\right): L \text { is a quadratic tower of } F \text { with } A_{L} \operatorname{split}\right\}\right\rangle=\operatorname{Nrd}\left(A^{*}\right) .
$$

In fact, for each $\lambda \in F^{*}$ there is a quadratic tower $L / F$ and $\alpha \in L^{*}$ such that $\lambda=N_{L / F}(\alpha)$.

Proof. By the classical norm principle for reduced norms, over any field we have the inclusion

$$
\left\langle\left\{N_{L / F}\left(L^{*}\right): L \text { is a quadratic tower of } F \text { with } A_{L} \operatorname{split}\right\}\right\rangle \subseteq \operatorname{Nrd}\left(A^{*}\right) .
$$

Thus to complete the proof, it suffices to show that under the assumption $I^{3}(F)=0$,

$$
F^{*} \subseteq\left\langle\left\{N_{L / F}\left(L^{*}\right): L \text { is a quadratic tower of } F \text { with } A_{L} \text { split }\right\}\right\rangle .
$$

Let $\exp (A)=2^{m}$. We prove the lemma by induction on $m$. Suppose $m=1$. Then $\exp (A)=2$ and hence by Merkurjev's Theorem [Me1], we write $A \sim H_{1} \otimes H_{2} \otimes$ $\cdots \otimes H_{r}$, where $r=r(A)$ and each $H_{i}$ is a quaternion algebra over $F$. We proceed further by induction on $r$. If $r=1$ the result holds by [BP2, Prop. 2.7]. Let $r \geq 2$ and $\lambda \in F^{*}$. By [BP2, Prop. 2.7] there exists a quadratic extension $L$ of $F$ which splits $H_{1}$ and $\lambda \in N_{L / F}\left(L^{*}\right)$. Then $r\left(A_{L}\right)<r$ and by 1.13 we have $I^{3}(L)=0$. Induction on $r$ leads to (1).

Suppose that $m \geq 2$. Then $\exp \left(A \otimes_{F} A\right)=2^{m-1}$. Let $\lambda \in F^{*}$. By induction, there exists a quadratic tower $L$ over $F$ and $\alpha \in L^{*}$ such that $\lambda=N_{L / F}(\alpha)$ and $\left(A \otimes_{F} A\right)_{L}$ is split. Then $\exp \left(A_{L}\right)=2$ and by $1.13, I^{3}(L)=0$. By the previous case, there exists a quadratic tower $M$ of $L$ with $\alpha \in N_{M / L}\left(M^{*}\right)$ and $A_{M}$ is split. 
Thus $M$ is a quadratic tower of $F$ such that $\lambda \in N_{M / F}\left(M^{*}\right)$ and $A_{M}$ is split. This completes the proof.

Proposition 2.2. Let $I^{3}(F)=0$ and let $Z$ be a quadratic extension of $F$. Let $A$ be a central simple algebra over $Z$ such that $\operatorname{cores}_{Z / F}([A])=0$ and $\exp (A)=2^{m}$. Then for each $\lambda \in F^{*}$, there exists a quadratic tower $L / F$ such that $\lambda \in N_{L / F}\left(L^{*}\right)$ and $A_{L}$ is split.

Proof. We prove this by induction on $m$. Suppose $m=1$. Since $\exp (A)=2$ and $\operatorname{cores}_{Z / F}([A])=0$, by 1.12 there exists a central simple algebra $A_{0}$ of exponent 2 over $F$ such that $A \sim A_{0} \otimes_{F} Z$. Let $\lambda \in F^{*}$. Since $I^{3}(F)=0$, by 2.1 , there exists a quadratic tower $L / F$ such that $\left(A_{0}\right)_{L}$ is split and $\lambda \in N_{L / F}\left(L^{*}\right)$. Clearly the extension $L$ splits $A$ and the proposition follows.

Suppose $m \geq 2$. Let $\lambda \in F^{*}$. Since $\exp \left(A \otimes_{Z} A\right)=2^{m-1}$, by induction there exists a quadratic tower $L / F$ such that $\lambda=N_{L / F}(\alpha)$ for some $\alpha \in L^{*}$, and $\left(A \otimes_{Z} A\right)_{L}$ splits. Clearly $\exp \left(A_{L}\right)=2$, and by the previous case we have a quadratic tower $M / L$ such that $A_{M}$ splits and $\alpha \in N_{M / L}\left(M^{*}\right)$. Then $M / F$ is a quadratic tower such that $\lambda \in N_{M / F}\left(M^{*}\right)$ and $A_{M}$ is split. This completes the proof.

We shall now describe norm principles for fields $F$ with $\operatorname{vcd}(F) \leq 2$. If $F$ has no orderings by $1.17, \operatorname{cd}(F) \leq 2$, and the results follow from the previous discussion. We shall assume in the rest of the section that $F$ has orderings. We denote by $\Omega$ the set of orderings on $F$. If $A$ is a central simple algebra over $F$, then $A$ is said to be locally split if $A \otimes_{F} F_{v}=A_{v}$ is split for each $v \in \Omega$.

Proposition 2.3. Let $\operatorname{vcd}(F) \leq 2$ and let $A$ be a central simple algebra over $F$ with $\exp (A)=2^{m}$. Then

$$
F^{*}=\left\langle\left\{N_{M / F}\left(M^{*}\right): M \in \mathcal{F}_{2}(F) \text { and } \operatorname{index}\left(A_{M}\right) \leq 2\right\}\right\rangle .
$$

Proof. We prove the proposition by induction on $m$. Let $m=1$. Then $\exp (A)=2$ and we proceed by further induction on $r(A)$. The statement is obvious if $r(A) \leq 1$. Let $r(A) \geq 2$ and $A \sim H_{1} \otimes H_{2} \otimes \cdots \otimes H_{r}$ with $r=r(A)$ and $H_{i}, 1 \leq i \leq r$, quaternion algebras over $F$. Let $H_{1}=(a, b)$ and $H_{2}=(c, d)$. Then to the algebra $H_{1} \otimes H_{2}$ is associated the Albert form ( $\left.c f .[\mathrm{KMRT}, \S 16 . \mathrm{A}]\right)$

$$
q=\langle-a,-b, a b, c, d,-c d\rangle .
$$

Since $\operatorname{disc}(q)=1$ and $\operatorname{dim}(q)=6$, the form $q$ is isotropic at $F_{v}$ for each $v \in \Omega$. Thus by $1.10, \operatorname{sn}(q)=F^{*}$ and by 1.5 we have

$$
F^{*}=\operatorname{sn}(q)=\left\langle\left\{N_{L / F}\left(L^{*}\right): L \text { is a quadratic extension of } F \text { and } q_{L} \text { is isotropic }\right\}\right\rangle \text {. }
$$

Let $L$ be a quadratic extension of $F$ with $q_{L}$ isotropic. By Albert's Theorem [KMRT, Th. 16.5], we have $r\left(\left(H_{1} \otimes H_{2}\right)_{L}\right) \leq 1$. Thus $r\left(A_{L}\right)<r(A)$ and by induction we have

$$
L^{*}=\left\langle\left\{N_{M / L}\left(M^{*}\right): M \in \mathcal{F}_{2}(L) \text { and } \operatorname{index}\left(A_{M}\right) \leq 2\right\}\right\rangle,
$$

and therefore taking norms from $L$ to $F$ we have

$$
\begin{aligned}
F^{*}=\operatorname{sn}(q) & =\left\langle\left\{N_{L / F}\left(L^{*}\right): L \text { is a quadratic extension of } F \text { and } q_{L} \text { is isotropic }\right\}\right\rangle \\
& \subseteq\left\langle\left\{N_{M / F}\left(M^{*}\right): M \in \mathcal{F}_{2}(F) \text { and } \operatorname{index}\left(A_{M}\right) \leq 2\right\}\right\rangle .
\end{aligned}
$$


This completes the case $m=1$. Now let $m \geq 2$. Then $\exp \left(A \otimes_{F} A\right)=2^{m-1}$ and by induction

$$
F^{*}=\left\langle\left\{N_{L / F}\left(L^{*}\right): L \in \mathcal{F}_{2}(F) \text { and } \operatorname{index}\left(\left(A \otimes_{F} A\right)_{L}\right) \leq 2\right\}\right\rangle .
$$

Let $L \in \mathcal{F}_{2}(F)$ be such that index $\left(\left(A \otimes_{F} A\right)_{L}\right) \leq 2$. Since the Brauer group of a real-closed field is isomorphic to $\mathbb{Z} / 2 \mathbb{Z}$, it follows that $\left(A \otimes_{F} A\right)_{L}$ is locally split. Thus by $1.8, \operatorname{Nrd}\left(\left(A \otimes_{F} A\right)_{L}\right)=L^{*}$. Since index $\left(\left(A \otimes_{F} A\right)_{L}\right) \leq 2$, we have

(3) $\operatorname{Nrd}\left(\left(A \otimes_{F} A\right)_{L}\right)$

$$
\subseteq\left\langle\left\{N_{N / L}\left(N^{*}\right): N \text { is a quadratic extension of } L \text { and }\left(A \otimes_{F} A\right)_{N} \text { is split }\right\}\right\rangle \text {. }
$$

Let $N$ be a quadratic extension of $L$ such that $\left(A \otimes_{F} A\right)_{N}$ is split. Then $\exp \left(A_{N}\right)=2$ and by the case $m=1$

$$
N^{*}=\left\langle\left\{N_{M / N}\left(M^{*}\right): M \in \mathcal{F}_{2}(N) \text { and } \operatorname{index}\left(\left(A_{M}\right) \leq 2\right\}\right\rangle .\right.
$$

Now it is clear from (2), (3) and (4) that

$$
F^{*}=\left\langle\left\{N_{M / F}\left(M^{*}\right): M \in \mathcal{F}_{2}(F) \text { and } \operatorname{index}\left(A_{M}\right) \leq 2\right\}\right\rangle .
$$

We refine 2.3 to the following:

Proposition 2.4. Let $F$ be a field with $\operatorname{vcd}(F) \leq 2$. Let $A$ be a central simple algebra over $F$ with $\exp (A)=2^{m}$ for some $m \geq 1$. Then,

$$
F^{*}=\left\langle\left\{N_{M / F}\left(M^{*}\right): M \in \mathcal{F}_{2}(F) \text { and } A_{M} \sim(-1,-x) \text { for some } x \in M^{*}\right\}\right\rangle .
$$

Proof. Let $L$ be a quadratic tower over $F$ such that $\operatorname{index}\left(A_{L}\right) \leq 2$. Then $A_{L} \sim$ $(a, b), a, b \in L^{*}$. Let $\Omega_{L}$ denote the set of orderings on $L$. For each $w \in \Omega_{L}$, the quadratic form $q^{\prime}=\langle-1,-a,-b, a b\rangle$ is isotropic over $L_{w}$, where $L_{w}$ denotes the real closure of $L$ at $w$. Therefore by [BP2, Prop. 7.7] we have $\operatorname{sn}\left(q^{\prime}\right)=L^{*}$. Thus, in view of 1.5 we have:

$$
L^{*}=\left\langle\left\{N_{M / L}\left(M^{*}\right): M \text { is a quadratic extension of } L \text { and } q_{M}^{\prime} \text { is isotropic }\right\}\right\rangle .
$$

Let $M$ be a quadratic extension of $L$ such that $q_{M}^{\prime}$ is isotropic. Then the form $\langle-a,-b, a b\rangle_{M}$ represents 1 , and we can write: $\langle-a,-b, a b\rangle_{M} \simeq\langle 1, x, y\rangle_{M}$; with $x, y \in M^{*}$. Comparing the discriminants, we have $\langle-a,-b, a b\rangle_{M} \simeq\langle 1, x, x\rangle_{M}$. Thus $\langle 1,-a,-b, a b\rangle_{M} \simeq\langle 1,1, x, x\rangle_{M}$ and $(a, b)_{M} \simeq(-1,-x)$. Thus,

$$
\begin{aligned}
L^{*} & =\left\langle\left\{N_{M / L}\left(M^{*}\right): M \text { is a quadratic extension of } F \text { and } q_{M}^{\prime} \text { is isotropic }\right\}\right\rangle \\
& \subseteq\left\langle\left\{N_{M / L}\left(M^{*}\right): M \in \mathcal{F}_{2}(L) \text { and } A_{M} \sim(-1,-x) \text { for some } x \in M^{*}\right\}\right\rangle
\end{aligned}
$$

and

$N_{L / F}\left(L^{*}\right) \subseteq\left\langle\left\{N_{M / F}\left(M^{*}\right): M \in \mathcal{F}_{2}(F)\right.\right.$ and $A_{M} \sim(-1,-x)$ for some $\left.\left.x \in M^{*}\right\}\right\rangle$.

This together with 2.3 gives

$$
F^{*} \subseteq\left\langle\left\{N_{M / F}\left(M^{*}\right): M \in \mathcal{F}_{2}(F) \text { and } A_{M} \sim(-1,-x) \text { for some } x \in M^{*}\right\}\right\rangle .
$$

This completes the proof.

Corollary 2.5. Let $\operatorname{vcd}(F) \leq 2$. Let $A_{1}$ and $A_{2}$ be central simple algebras over $F$ with $\exp \left(A_{i}\right)$ a power of 2 for $i=1,2$. Then we have:

$$
\begin{gathered}
F^{*}=\left\langle\left\{ N_{M / F}\left(M^{*}\right): M \in \mathcal{F}_{2}(F) \text { and } A_{1 M} \sim(-1,-x),\right.\right. \\
\left.\left.A_{2 M} \sim(-1,-y) \text { for some } x, y \in M^{*}\right\}\right\rangle .
\end{gathered}
$$


The following is a refinement of the surjectivity of the reduced norm (Theorem 1.8) for locally split algebras with centre a quadratic extension of $F$.

Proposition 2.6. Let $\operatorname{vcd}(F) \leq 2$ and let $F$ have orderings. Let $\Omega$ denote the set of orderings on $F$. Let $Z=F(\sqrt{\delta})$ be a quadratic extension of fields. Let $A$ be a central simple $Z$-algebra which is split at each $v \in \Omega$. Further assume that $\exp (A)=2^{m}$ for some integer $m$ and $\operatorname{cores}_{Z / F}(A)=0$. Then for each $\lambda \in F^{*}$, there exist extensions $E_{i}$ over $F$ and $\lambda_{i} \in E_{i}^{*}$ such that each $A \otimes_{F} E_{i}$ is split and $\lambda=\prod_{i} N_{E_{i} / F}\left(\lambda_{i}\right)$.

Proof. We proceed by induction on $m$. Let $m=1$. Since $\operatorname{cores}_{Z / F}(A)=0$, by 1.12 there is a central simple algebra $A_{0}$ over $F$ with $\exp \left(A_{0}\right)=2$ and $A \sim A_{0} \otimes_{F} Z$. By [Me1], there are quaternion algebras $H_{i} ; 1 \leq i \leq r=r\left(A_{0}\right)$ over $F$ such that $A_{0} \sim H_{1} \otimes H_{2} \otimes \cdots \otimes H_{r}$. Suppose $r=1$. Then $A_{0} \sim H_{1}=(a, b)$ for some $a, b \in F^{*}$. Let $q$ denote the quadratic form $\langle 1,-a,-b, a b \delta\rangle$ over $F$. Then by [CTSk, Prop. 2.3] we have $\operatorname{sn}(q)=\operatorname{Nrd}\left(\left(H_{1} \otimes_{F} F(\sqrt{\delta})\right)^{*}\right) \cap F^{*}$. Since $A$ is locally split, by $1.8 \operatorname{Nrd}\left(\left(H_{1} \otimes_{F} F(\sqrt{\delta})\right)^{*}\right)=\operatorname{Nrd}\left(A^{*}\right)=Z^{*}$. Therefore $\operatorname{sn}(q)=F^{*}$. Thus by 1.5, for each $\lambda \in F^{*}$, there exist quadratic extensions $E_{i} / F$ and $\lambda_{i} \in E_{i}^{*}$ such that each $q_{E_{i}}$ is isotropic and $\lambda=\prod_{i} N_{E_{i} / F}\left(\lambda_{i}\right)$. Further $A \otimes_{F} E_{i} \sim(a, b) \otimes_{E_{i}} E_{i}(\sqrt{\delta})$ and the norm form of $(a, b) \otimes_{E_{i}} E_{i}(\sqrt{\delta})$ is isometric to $q_{E_{i}(\sqrt{\delta})}$, which is isotropic. It follows therefore that each $A \otimes_{F} E_{i}$ is split. Thus $F^{*}$ is generated by the norms from those extensions of $F$ where the algebra $A$ is split.

Now suppose $r \geq 2$. Then by 2.3 we have

$$
F^{*}=\left\langle\left\{N_{L / F}\left(L^{*}\right): \operatorname{index}\left(\left(A_{0}\right)_{L}\right) \leq 2\right\}\right\rangle .
$$

The proposition follows immediately from the case $r=1$.

Let $m \geq 2$. Then $\exp \left(A \otimes_{Z} A\right)=2^{m-1}$ and at each $v \in \Omega$ the algebra

$$
\left(A \otimes_{Z} A\right) \otimes_{F} F_{v}
$$

is split since $\operatorname{Br}\left(F_{v}\right)=\mathbb{Z} / 2 \mathbb{Z}$. Thus by induction, $F^{*}$ is generated by norms from extensions $M_{i}$ over $F$ such that the algebra $\left(A \otimes_{Z} A\right) \otimes_{F} M_{i}$ splits. It is clear that $\exp \left(A \otimes_{F} M_{i}\right)=2$. Thus by the exponent 2 case, it follows that each $M_{i}^{*}$ is generated by norms from extensions $E_{i}$ of $M_{i}$ such that $A \otimes_{F} E_{i}$ is split. We conclude therefore, that $F^{*}$ is generated by norms from those extensions of $F$ where $A$ splits.

\section{Fields With $\operatorname{cd}(F) \leq 2$}

In this section, we prove that if $\operatorname{cd}(F) \leq 2$, then for adjoint classical groups $G$ of type ${ }^{2} A_{n}, C_{n}$ and $D_{n}, G(F) / R=0$. We begin with the result leading to the triviality of $G(F) / R$ in the $C_{n}$ case.

Theorem 3.1. Let $F$ be a field with $\operatorname{char}(F) \neq 2$ and $I^{3}(F)=0$. Let $A$ be a central simple algebra of degree $2 n$ over $F$ and let $\sigma$ be a symplectic involution on A. Then $\operatorname{Hyp}(A, \sigma)=F^{*}$.

Proof. Let $\lambda \in F^{*}$. Since exponent of $A$ is 2 and $I^{3}(F)=0$, by 2.1 , there exists a quadratic tower $L / F$ such that $L$ splits $A$ and $\lambda \in N_{L / F}\left(L^{*}\right)$. The involution $\sigma_{L}$ is adjoint to a skew-symmetric form $h_{L}$ over $L$ which is hyperbolic. Therefore $\lambda \in \operatorname{Hyp}(A, \sigma)$. 
Let $q$ be a quadratic form over $F$ of rank $2 n$. Let $\sigma$ be the involution on $M_{2 n}(F)$ which is adjoint to $q$. We denote by $C(q)$ the Clifford invariant of $q$.

Proposition 3.2. If $I^{3}(F)=0$, then $G(q) \subseteq \operatorname{Hyp}(q)$.

Proof. We first assume that the discriminant of $q$ is trivial. Let $\lambda \in F^{*}$. The algebra $C(q)$ has exponent 2 and by 2.1, there exists a quadratic tower $M$ of $F$ such that $C(q) \otimes_{F} M$ is split and $\lambda \in N_{M / F}\left(M^{*}\right)$. By $1.13, I^{3}(M)=0$. Since $q_{M}$ is an even dimensional quadratic form with trivial discriminant and trivial Clifford invariant, in view of [EL, Th. 3] $q_{M}$ is hyperbolic and hence $\lambda \in \operatorname{Hyp}(q)$. Thus $\operatorname{Hyp}(q)=F^{*}$.

Now suppose that $\operatorname{disc}(q)$ is non-trivial, $d \in F^{*}$ is a representative of the square class of $\operatorname{disc}(q)$ in $F^{*} / F^{* 2}$ and $L=F(\sqrt{d})$. Let $\lambda \in G(q)$. By 1.2, $\lambda \in N_{L / F}\left(L^{*}\right)$. Since $\operatorname{disc}\left(q_{L}\right)=1$, by the previous case $L^{*}=\operatorname{Hyp}\left(q_{L}\right)$. Taking norms we get $N_{L / F}\left(L^{*}\right) \subseteq \operatorname{Hyp}(q)$. Thus $G(q) \subseteq \operatorname{Hyp}(q)$.

We prove a similar result when $A$ is not split.

Theorem 3.3. Let $I^{3}(F)=0$. Let $A$ be a central simple algebra with an involution $\sigma$ of orthogonal type. Let $d$ be the discriminant of $\sigma$ and let $L=F[X] /\left(X^{2}-d\right)$. Then $G_{+}(A, \sigma)=\operatorname{Hyp}(A, \sigma)=N_{L / F}\left(L^{*}\right)$.

Proof. Since $A$ supports an involution of first kind, $\exp (A) \leq 2$. Suppose first that $\operatorname{disc}(\sigma)$ is trivial. Let $M$ be a quadratic tower of $F$ which splits $A$. By the proof of 3.2 we have $M^{*}=\operatorname{Hyp}\left(A_{M}, \sigma_{M}\right)$. Thus $N_{M / F}\left(M^{*}\right) \subseteq \operatorname{Hyp}(A, \sigma)$. This, together with 2.1 implies that $F^{*}=\operatorname{Nrd}\left(A^{*}\right) \subseteq \operatorname{Hyp}(A, \sigma)$. Hence $F^{*}=$ $\operatorname{Hyp}(A, \sigma)=G_{+}(A, \sigma)$. Since $L=F \times F$, we have $N_{L / F}\left(L^{*}\right)=F^{*}$. Thus $G_{+}(A, \sigma)=\operatorname{Hyp}(A, \sigma)=N_{L / F}\left(L^{*}\right)$.

Suppose that $\operatorname{disc}(\sigma)$ is not trivial. Let $d \in F^{*}$ represent the class of $\operatorname{disc}(\sigma)$ in $F^{*} / F^{* 2}$. Let $\lambda \in G_{+}(A, \sigma)$. Then by 1.3 , we have $\lambda \in N_{L / F}\left(L^{*}\right)$ where $L=$ $F(\sqrt{d})$. Clearly $\operatorname{disc}\left(\sigma_{L}\right)=1$ and by the previous case $L^{*}=\operatorname{Hyp}\left(A_{L}, \sigma_{L}\right)$. Thus $\lambda \in N_{L / F}\left(L^{*}\right) \subseteq \operatorname{Hyp}(A, \sigma)$. Thus $G_{+}(A, \sigma) \subseteq N_{L / F}\left(L^{*}\right) \subseteq \operatorname{Hyp}(A, \sigma)$. By 1.1, $\operatorname{Hyp}(A, \sigma) \cdot F^{* 2} \subseteq G_{+}(A, \sigma)$. Hence $G_{+}(A, \sigma)=\operatorname{Hyp}(A, \sigma)=N_{L / F}\left(L^{*}\right)$.

Let $Z$ be a quadratic extension of $F$ and let $A$ be a central simple algebra over $Z$ with an involution $\sigma$ of the second kind such that $Z^{\sigma}=F$. In the next lemma, we consider the case where $A$ splits and the involution $\sigma$ is adjoint to a $Z / F$-hermitian form $h$. In view of 1.6, we further assume that $h$ has even rank; i.e. $\operatorname{deg}(A)$ is even.

Lemma 3.4. Let $I^{3}(F)=0$, let $A$ be split and let $\sigma$ be an involution of the second kind on $A$ such that $Z^{\sigma}=F$. Then $\operatorname{Hyp}(A, \sigma)=F^{*}$.

Proof. Let $Z=F(\sqrt{\delta})$. Let $q_{h}$ be the quadratic form over $F$ defined by $q_{h}(x)=$ $h(x, x)$. Then $q_{h} \simeq\langle 1,-\delta\rangle \otimes q$ [Sc, pp. 349, Remark 1.3] for some quadratic form $q$ over $F$ having the same rank as $h$, which is even. Therefore $q_{h} \in I^{2}(F)$ and by a theorem of Jacobson [MH, pp. 114], the form $h$ is hyperbolic over an extension $M$ of $F$ if and only if the quadratic form $q_{h}$ is hyperbolic over $M$. Let $C$ denote the Clifford algebra of $q_{h}$. Let $\lambda \in F^{*}$. By 2.1, there exists a quadratic tower $M$ over $F$ such that $C_{M}$ is split and $\lambda \in N_{M / L}\left(M^{*}\right)$. Since $I^{3}(M)=0$, by [EL, Th. 3], $\left(q_{h}\right)_{M}$ is hyperbolic and hence the hermitian form $h_{M}$ is hyperbolic. Therefore $N_{M / F}\left(M^{*}\right) \subseteq \operatorname{Hyp}(A, \sigma)$. Thus $\operatorname{Hyp}(A, \sigma)=F^{*}$.

Theorem 3.5. If $I^{3}(F)=0$ and $\exp (A)=2^{m}$, then $\operatorname{Hyp}(A, \sigma)=F^{*}$. 
Proof. Since $A$ supports an involution $\sigma$ of the second kind, by [Sc, Th. 9.5] we have $\operatorname{cores}_{Z / F}(A)=0$. Therefore by 2.2 , given $\lambda \in F^{*}$ there exists a quadratic tower $L / F$ such that $A_{L}$ splits and $\lambda \in N_{L / F}\left(L^{*}\right)$. Since $A_{L}$ is split, by 3.4, $L^{*}=\operatorname{Hyp}\left(A_{L}, \sigma_{L}\right)$. Taking norms we conclude that $\lambda \in \operatorname{Hyp}(A, \sigma)$. Therefore $\operatorname{Hyp}(A, \sigma)=F^{*}$.

Theorem 3.6. Let $\operatorname{cd}(F) \leq 2$ and let $Z$ be a quadratic extension of $F$. Let $A$ be a central simple algebra of even degree over $Z$ with an involution $\sigma$ of the second kind such that $Z^{\sigma}=F$. Then $\operatorname{Hyp}(A, \sigma) \cdot F^{* 2}=F^{*}$.

Proof. By [BP1, Lemma 3.3.1], there exists an odd degree extension $L$ over $F$ such that $\exp \left(A \otimes_{F} L\right)$ is a power of 2 . Since the condition $\operatorname{cd}(F) \leq 2$ is preserved under finite extensions of fields [Ar, Th 2.1], we have $\operatorname{cd}(L) \leq 2$. By $1.14 I^{3}(L)=0$, and by $3.5 \operatorname{Hyp}\left(A_{L}, \sigma_{L}\right)=L^{*}$. Hence $N_{L / F}\left(L^{*}\right) \subseteq \operatorname{Hyp}(A, \sigma)$. Let $\lambda \in F^{*}$ and let $[L$ : $F]=2 s+1$. Then $\lambda^{2 s+1}=N_{L / F}(\lambda) \in \operatorname{Hyp}(A, \sigma)$ and we have $\lambda \in \operatorname{Hyp}(A, \sigma) . F^{* 2}$. This implies that $\operatorname{Hyp}(A, \sigma) \cdot F^{* 2}=F^{*}$.

Theorem 3.7. If $\operatorname{cd}(F) \leq 2$ and $G$ an adjoint group of classical type defined over $F$, then $G(F) / R=0$.

Proof. A classical adjoint group $G$ is a direct product of groups $R_{L_{i} / F}\left(G_{i}\right)$, where $L_{i} / F$ are finite extensions and $G_{i}$ are absolutely simple adjoint groups of classical type defined over $L_{i}[\mathrm{~T}, 3.1 .2]$. Moreover, $G_{i}\left(L_{i}\right) / R=R_{L_{i} / F}\left(G_{i}\right)(F) / R$ and $R$ equivalence commutes with direct products [CTS, pp. 195]. In view of this, it suffices to prove the theorem for an absolutely simple classical adjoint group $G$ defined over $F$. By [We] such an algebraic group is isomorphic to $\mathbf{P S i m}_{+}(A, \sigma)$ for a central simple algebra $A$ over a field $Z,[Z: F] \leq 2$, with an involution $\sigma$. In view of 1.1 and 1.14, the result follows in the ${ }^{2} A_{n}$ case from 3.6 and 1.6 , in the $B_{n}$ case from 1.4, in the $C_{n}$ case from 3.1 and 1.7, and in the $D_{n}$ case from 3.3.

Remark. Theorem 3.7 for groups of types $A_{n}$ and $C_{n}$ also follows from [CTGP, Cor. 4.11], using the fact that $G(F) / R=0$ if $G$ is simply connected of type $A_{n}$ or $C_{n}$, and [G, pp. 222].

\section{Fields with $\operatorname{vcd}(F) \leq 2$ : Symplectic Groups}

In this section $F$ denotes a formally real field with $\operatorname{vcd}(F) \leq 2$, and $\Omega$ the set of orderings on $F$. Let $A$ be a central simple algebra over $F$ of degree $2 n$ and let $\sigma$ be an involution of symplectic type on $A$. In view of 1.7 , we assume that $n$ is even. We say that $\sigma$ is locally hyperbolic if for each $v \in \Omega$, the involution $\sigma_{v}$ on $A_{v}=A \otimes_{F} F_{v}$ is hyperbolic, $F_{v}$ denoting the real closure of $F$ at $v$.

Proposition 4.1. Let $A$ be a central simple algebra over $F$ of degree $2 n$, where $n$ is an even integer. Let $\sigma$ be a symplectic involution on A. If $\sigma$ is locally hyperbolic, then $\operatorname{Hyp}(A, \sigma)=F^{*}$.

Proof. First assume that $A=M_{n}(H)$, where $H$ is a quaternion algebra over $F$. Let bar denote the canonical involution on $H$ and $h$ a hermitian form of rank $n$ over $(H,-)$ such that $\sigma$ is adjoint to $h$. Since $\sigma$ is locally hyperbolic, so is $h$ and hence $\operatorname{sgn}(h)=0$. Thus $h$ has even rank and trivial signature, and by [BP2, Th. 6.2], the form $h$ itself is hyperbolic. $\operatorname{Thus} \operatorname{Hyp}(A, \sigma)=F^{*}$. 
Suppose $A$ is arbitrary. Since $A$ supports an involution, $\exp (A)=2[\mathrm{Sc}$, Th. 8.4 ] and by 2.3 , we have

$$
F^{*}=\left\langle\left\{N_{M / F}\left(M^{*}\right): \operatorname{index}\left(A_{M}\right) \leq 2\right\}\right\rangle .
$$

Let $M$ be a finite extension of $F$ such that index $\left(A_{M}\right) \leq 2$. Then $A_{M} \simeq M_{n}(H)$ where $H$ is a quaternion algebra over $M$. Since $\sigma$ is locally hyperbolic, so is $\sigma_{M}$, and by the previous case, $M^{*}=\operatorname{Hyp}\left(A_{M}, \sigma_{M}\right)$. Therefore $N_{M / F}\left(M^{*}\right) \subseteq \operatorname{Hyp}(A, \sigma)$ and in view of $(*)$ we get $\operatorname{Hyp}(A, \sigma)=F^{*}$.

Theorem 4.2. Let $F$ be a formally real field with $\operatorname{vcd}(F) \leq 2$. Let $A$ be a central simple algebra over $F$ of degree $2 n$ and let $\sigma$ be a symplectic involution on $A$. Then $G(A, \sigma) \subseteq \operatorname{Hyp}(A, \sigma)$.

Proof. In view of 1.7, we assume that $n$ is even. Let $\lambda \in G(A, \sigma)$ and $K=F(\sqrt{-\lambda})$. Let $\Omega_{K}$ denote the set of orderings on $K$. For each $w \in \Omega_{K}, \lambda \equiv-1$ modulo $K_{w}^{* 2}$ is a similarity factor for $\sigma_{K}$, and hence $\operatorname{sgn}\left(\sigma_{K}\right)=0$. Further $\operatorname{deg}(A)$ is divisible by 4 and hence the involution $\sigma_{K}$ is locally hyperbolic. Thus by 4.1 , we have $\operatorname{Hyp}\left(A_{K}, \sigma_{K}\right)=K^{*}$. Therefore

$$
\lambda=N_{K / F}(\sqrt{-\lambda}) \in N_{K / F}\left(K^{*}\right)=N_{K / F}\left(\operatorname{Hyp}\left(A_{K}, \sigma_{K}\right)\right) \subseteq \operatorname{Hyp}(A, \sigma) .
$$

\section{Fields With $\operatorname{vcd}(F) \leq 2$ : Unitary GROUpS}

Let $F$ be an arbitrary field with $\operatorname{char}(F) \neq 2$. Let $Z=F(\sqrt{\delta})$ be a quadratic extension of $F$. Let $A$ be a central simple algebra over $Z$ and let $\sigma$ be an involution on $A$ such that $Z^{\sigma}=F$. In view of 1.6 , we assume throughout this section that $A$ has even degree.

Let $\operatorname{deg}(A)=2 m$ and $D=D(A, \sigma)$ denote the discriminant algebra of $(A, \sigma)$ (cf. [KMRT, §10.E]). The algebra $D$ is a central simple algebra over $F$ and carries an involution $\bar{\sigma}$ of the first kind, which is of symplectic type if $m$ is odd and of orthogonal type if $m$ is even [KMRT, Prop. 10.30]. For $1 \leq i \leq 2 m$, let $\bigwedge^{i} A$ be the $i^{\text {th }}$ exterior power of $A$ (cf. [KMRT, $\left.\S 10(10.4)\right]$ ). By [KMRT, Prop. 14.3], there is a homogeneous polynomial map $\bigwedge^{i}: A \rightarrow \bigwedge^{i} A$ of degree $i, 1 \leq i \leq 2 \mathrm{~m}$. If $A=\operatorname{End}_{F}(V)$, then $\bigwedge^{i} A=\operatorname{End}_{F}\left(\Lambda^{i} V\right)$ and $\bigwedge^{i}(f)=\Lambda^{i}(f)$, the $i^{\text {th }}$ exterior power of the linear map $f \in \operatorname{End}_{F}(V)$.

Theorem 5.1. Let $F$ be a field with $\operatorname{char}(F) \neq 2$. Let $A$ be a central simple algebra of degree $2 m$ over a field $Z$ with $m$ odd. Let $\sigma$ be an involution of the second kind on $A$ such that $Z^{\sigma}=F$. Let $D=D(A, \sigma)$ be the discriminant algebra of $(A, \sigma)$. Then $G(A, \sigma) \subseteq \operatorname{Nrd}\left(D^{*}\right) \cdot N_{Z / F}\left(Z^{*}\right)$.

Proof. Let $x \in G(A, \sigma)$ and $g \in \operatorname{Sim}(A, \sigma)$ be such that $\mu(g)=\sigma(g) g=x$. Then $N_{Z / F}(\operatorname{Nrd}(g))=\mu(g)^{2 m}$ and by Hilbert Theorem-90, there exists $\alpha \in Z^{*}$ such that $\mu(g)^{-m} \operatorname{Nrd}(g)=\alpha^{-1} \bar{\alpha}$, where bar denotes the non-trivial automorphism of $Z$ over $F$. By [KMRT, Lemma 14.6], we have

$$
\bar{\sigma}\left(\alpha^{-1} \wedge^{m} g\right) \alpha^{-1} \wedge^{m} g=N_{Z / F}(\alpha)^{-1} \mu(g)^{m} .
$$

Since $m$ is odd, $x=\mu(g) \in G(D, \bar{\sigma}) \cdot N_{Z / F}\left(Z^{*}\right)$. Thus

$$
G(A, \sigma) \subseteq G(D, \bar{\sigma}) \cdot N_{Z / F}\left(Z^{*}\right) .
$$


Let $y \in G(D, \bar{\sigma})$ be arbitrary and let $h \in \operatorname{Sim}(D, \bar{\sigma})$ be such that $\mu(h)=\bar{\sigma}(h) h=$ $y$. Since $m$ is odd, the involution $\bar{\sigma}$ is of symplectic type and by [KMRT, Prop. 12.23] we have $\mu(h)^{m}=\operatorname{Nrd}(h)$. Again, since $m$ is odd, we have $y=\mu(h) \in$ $\operatorname{Nrd}\left(D^{*}\right) \cdot F^{* 2}$. Thus

$$
G(D, \bar{\sigma}) \subseteq \operatorname{Nrd}\left(D^{*}\right) \cdot F^{* 2}
$$

and combining the inclusions $(*)$ and $(* *)$ above, we get

$$
G(A, \sigma) \subseteq \operatorname{Nrd}\left(D^{*}\right) \cdot N_{Z / F}\left(Z^{*}\right) .
$$

This completes the proof.

In this section, from now onwards we assume that $\operatorname{vcd}(F) \leq 2, F$ has orderings and denote by $\Omega$ the set of orderings on $F$. A quadratic form $q$ over $F$ is called locally hyperbolic if $q$ is hyperbolic at every real closure $F_{v}, v \in \Omega$.

Lemma 5.2. If $q$ is a locally hyperbolic quadratic form of even rank and trivial discriminant over $F, \operatorname{Hyp}(q)=F^{*}$.

Proof. Since $q$ is locally hyperbolic the Clifford algebra $C(q)$ of $q$ is locally split. Thus by 1.8 we have $\operatorname{Nrd}\left(C(q)^{*}\right)=F^{*}$. Let $\lambda \in F^{*}$ and let $L / F$ be a finite extension such that $\lambda \in N_{L / F}\left(L^{*}\right)$ and $C(q)_{L}$ is split. Then $q_{L}$ has even dimension, trivial discriminant, trivial Clifford invariant and $\operatorname{sgn}\left(q_{L}\right)=0$. Therefore by [EL, Th. 3], the form $q_{L}$ is hyperbolic and $\lambda \in N_{L / F}\left(L^{*}\right) \in \operatorname{Hyp}(q)$.

Proposition 5.3. Let $Z=F(\sqrt{\delta})$ be a quadratic extension. Let $A=M_{r}(Z)$, where $r$ is an even positive integer, support a locally hyperbolic $Z / F$-involution $\sigma$. Then $\operatorname{Hyp}(A, \sigma)=F^{*}$.

Proof. Let the involution $\sigma$ be adjoint to a $Z / F$-hermitian form $h$. Then the rank of $h$ is $r$. Let $q_{h}$ be the quadratic form over $F$ given by $q_{h}(x)=h(x, x)$. Then $q_{h} \simeq\langle 1,-\delta\rangle \otimes q$ [Sc, pp. 349, Remark 1.3], where $q$ is a quadratic form over $F$ of the same rank as that of $h$, which is even. Therefore $q_{h} \in I^{2}(F)$. By Jacobson's theorem [MH, p. 114], the form $h_{M}$ is hyperbolic if and only if the quadratic form $\left(q_{h}\right)_{M}$ is hyperbolic. It follows that $\operatorname{Hyp}(A, \sigma)=\operatorname{Hyp}\left(q_{h}\right)$. Since $h$ is locally hyperbolic, the form $q_{h}$ is locally hyperbolic as well. By 5.2 , we have $\operatorname{Hyp}\left(q_{h}\right)=F^{*}$. $\operatorname{Thus} \operatorname{Hyp}(A, \sigma)=\operatorname{Hyp}\left(q_{h}\right)=F^{*}$.

The following is a consequence of 5.3 and 2.6.

Proposition 5.4. Let $A$ be a locally split central simple $Z$-algebra and let $\sigma$ be a locally hyperbolic $Z / F$-involution on $A$. Let $\exp (A)=2^{m}$. Then $\operatorname{Hyp}(A, \sigma)=F^{*}$.

Proof. Since $A$ supports an involution $\sigma$ of the second kind with $Z^{\sigma}=F$, by [Sc, Th. 9.5], $\operatorname{cores}_{Z / F}(A)=0$. Thus by 2.6 we have

$$
F^{*}=\left\langle\left\{N_{L / F}\left(L^{*}\right): A_{L} \text { is split }\right\}\right\rangle .
$$

Let $L / F$ be an extension which splits $A$. By 5.3, $\operatorname{Hyp}\left(A_{L}, \sigma_{L}\right)=L^{*}$, and taking norm from $L / F$, we conclude that $\operatorname{Hyp}(A, \sigma)=F^{*}$.

Proposition 5.5. Let $A$ be a central simple algebra over $Z$ of degree $2 m$, where $m$ is odd. Let $\sigma$ be a $Z / F$-involution on $A$ with $\operatorname{sgn}(\sigma)=0$. Let $D=D(A, \sigma)$ be the discriminant algebra of $(A, \sigma)$. Then $\operatorname{Nrd}\left(D^{*}\right) \subseteq \operatorname{Hyp}(A, \sigma) . F^{* 2}$. Further

$$
G(A, \sigma)=\operatorname{Nrd}\left(D^{*}\right) \cdot N_{Z / F}\left(Z^{*}\right)=\operatorname{Hyp}(A, \sigma) \cdot N_{Z / F}\left(Z^{*}\right) .
$$


Proof. We first show that $\operatorname{Nrd}\left(D^{*}\right) \subseteq \operatorname{Hyp}(A, \sigma) \cdot F^{* 2}$. Assume first that $\exp (A)$ is a power of 2. Since $\operatorname{deg}(A)=2 m$ with $m$ odd, $\operatorname{index}(A)=\exp (A)=2$ and $A=M_{m}(H)$ for some quaternion algebra $H$ over $Z$. By [KMRT, §10.4], [KMRT, Prop. 10.30] and the hypothesis that $m$ is odd, it follows that

$$
D \otimes_{F} Z \simeq \bigwedge^{m}\left(M_{m}(H)\right) \sim H^{\otimes m} \sim H .
$$

Thus if $M$ is a finite extension of $F$ such that $D_{M}$ is split, then $H_{M}$ is split and $\operatorname{sgn}\left(\sigma_{M}\right)=0$. Thus by $5.3, \operatorname{Hyp}\left(A_{M}, \sigma_{M}\right)=M^{*}$ and taking norms, $N_{M / F}\left(M^{*}\right) \subseteq$ $\operatorname{Hyp}(A, \sigma)$. In view of the classical norm principle for reduced norms, $\operatorname{Nrd}\left(D^{*}\right) \subseteq$ $\operatorname{Hyp}(A, \sigma)$.

Now suppose that $\exp (A)$ is arbitrary. By [BP1, Lemma 3.3.1], there exists an odd degree extension $L / F$ such that $\exp \left(A_{L}\right)$ is a power of 2. Let $\lambda \in \operatorname{Nrd}\left(D^{*}\right)$. Then $\lambda \in \operatorname{Nrd}\left(D_{L}^{*}\right)$. By the previous case, $\lambda \in \operatorname{Hyp}\left(A_{L}, \sigma_{L}\right)$. Taking norm from $L / F$ and using the hypothesis that $m$ is odd, we conclude that $\lambda \in \operatorname{Hyp}(A, \sigma) . F^{* 2}$. This proves the first assertion of 5.5. It follows immediately that

$$
\operatorname{Nrd}\left(D^{*}\right) \cdot N_{Z / F}\left(Z^{*}\right) \subseteq \operatorname{Hyp}(A, \sigma) \cdot N_{Z / F}\left(Z^{*}\right) .
$$

From 5.1, it is clear that

$$
G(A, \sigma) \subseteq \operatorname{Nrd}\left(D^{*}\right) \cdot N_{Z / F}\left(Z^{*}\right)
$$

and further by $1.1, \operatorname{Hyp}(A, \sigma) \cdot N_{Z / F}\left(Z^{*}\right) \subseteq G(A, \sigma)$. In view of this and the inclusions $(*)$ and $(* *)$ we conclude that

$$
G(A, \sigma)=\operatorname{Nrd}\left(D^{*}\right) \cdot N_{Z / F}\left(Z^{*}\right)=\operatorname{Hyp}(A, \sigma) \cdot N_{Z / F}\left(Z^{*}\right) .
$$

Theorem 5.6. Let $F$ be a field with $\operatorname{vcd}(F) \leq 2$ and let $Z$ be a quadratic extension of $F$. Let $A$ be a central simple algebra over $Z$ of degree $2 m$, where $m$ is odd. Let $\sigma$ be a $Z / F$-involution on $A$. Then $G(A, \sigma)=\operatorname{Hyp}(A, \sigma) \cdot N_{Z / F}\left(Z^{*}\right)$.

Proof. Let $\lambda \in G(A, \sigma)$. Let $D=D(A, \sigma)$ be the discriminant algebra of $(A, \sigma)$. By 5.1, $\lambda \in \operatorname{Nrd}\left(D^{*}\right) . N_{Z / F}\left(Z^{*}\right)$. Let $\lambda_{1} \in \operatorname{Nrd}\left(D^{*}\right)$ and $\alpha \in N_{Z / F}\left(Z^{*}\right)$ be such that $\lambda=\lambda_{1} \alpha$. Since $N_{Z / F}\left(Z^{*}\right) \subseteq \operatorname{Hyp}(A, \sigma) \subseteq G(A, \sigma)$, it follows that $\alpha \in G(A, \sigma)$. Let $K=F\left(\sqrt{-\lambda_{1}}\right)$. Then $\operatorname{sgn}\left(\sigma_{K}\right)=0$ and by $5.5, \operatorname{Nrd}\left(D_{K}^{*}\right) \subseteq \operatorname{Hyp}\left(A_{K}, \sigma_{K}\right) \cdot K^{* 2}$. Further, since $\lambda_{1} \in \operatorname{Nrd}\left(D_{K}^{*}\right)$ and $\lambda_{1} \equiv-1 \bmod K^{* 2}, D_{K}$ is locally split, and by $1.8, \operatorname{Nrd}\left(D_{K}^{*}\right)=K^{*}$. Thus $\operatorname{Hyp}\left(A_{K}, \sigma_{K}\right) \cdot K^{* 2}=K^{*}$. Taking norms, we get

$$
\lambda_{1} \in N_{K / F}\left(K^{*}\right)=N_{K / F}\left(\operatorname{Hyp}\left(A_{K}, \sigma_{K}\right)\right) \subseteq \operatorname{Hyp}(A, \sigma) .
$$

Thus $\lambda=\lambda_{1} \alpha \in \operatorname{Hyp}(A, \sigma) \cdot N_{Z / F}\left(Z^{*}\right)$. This completes the proof. $F$.

Let $\Sigma(F)$ denote the set of elements of $F$ which are positive at all orderings of

Lemma 5.7. Let $\alpha, \delta \in F^{*}$. Then we have:

$$
\begin{aligned}
& F^{*}=\left\langle\left\{ N_{L / F}\left(L^{*}\right): L / F\right.\right. \text { is a quadratic extension such that there exists } \\
& \left.\left.\qquad u_{L} \in L(\sqrt{\delta}) \text { with } N_{L(\sqrt{\delta}) / L}\left(u_{L}\right)=1 \text { and } \alpha u_{L} \in \Sigma(L(\sqrt{\delta}))\right\}\right\rangle .
\end{aligned}
$$

Proof. Since the quadratic form $\phi=\langle 1, \delta,-\alpha, \delta \alpha\rangle$ is locally isotropic, by 1.5 and 1.10 ,

$(*)$ $F^{*}=\operatorname{sn}(\phi)=\left\langle\left\{N_{L / F}\left(L^{*}\right): L / F\right.\right.$ is a quadratic extension and $\phi_{L}$ is isotropic $\left.\}\right\rangle$. 
At an extension $L / F$ where $\phi$ is isotropic, we choose $a, b, c, d \in L^{*}$ such that $a^{2}+\delta b^{2}-\alpha c^{2}+\delta \alpha d^{2}=0$. If $c^{2}+\delta d^{2}=0$ or $a^{2}+\delta b^{2}=0$, clearly $L(\sqrt{\delta})$ has no ordering and thus $\Sigma(L(\sqrt{\delta}))=L(\sqrt{\delta})^{*}$. In this case, we may take $u_{L}=$ 1. Otherwise, we let $\theta=c+d \sqrt{\delta}$ and $u_{L}=\theta^{-1} \bar{\theta}$, where $\bar{\theta}=c-d \sqrt{\delta}$. It is immediate that $\operatorname{Tr}_{L(\sqrt{\delta}) / L}\left(u_{L}\right)=2\left(c^{2}+\delta d^{2}\right)\left(c^{2}-\delta d^{2}\right)^{-1}$ and $N_{L(\sqrt{\delta}) / L}\left(u_{L}\right)=1$. Since $a^{2}+\delta b^{2}-\alpha c^{2}+\delta \alpha d^{2}=0$ and both $c^{2}+\delta d^{2}$ and $c^{2}-\delta d^{2}$ are units, it follows that

$$
\alpha=\left(\left(a^{2}+\delta b^{2}\right)\left(c^{2}+\delta d^{2}\right)^{-1}\right)\left(\left(c^{2}+\delta d^{2}\right)\left(c^{2}-\delta d^{2}\right)^{-1}\right)
$$

Thus

$2 \alpha \operatorname{Tr}_{L(\sqrt{\delta}) / L}\left(u_{L}\right)=\left(a^{2}+\delta b^{2}\right)\left(c^{2}+\delta d^{2}\right)^{-1}\left(\operatorname{Tr}_{L(\sqrt{\delta}) / L}\left(u_{L}\right)\right)^{2} \in N_{L(\sqrt{-\delta}) / L}(L(\sqrt{-\delta}))$

and hence the quaternion algebra $\left(2 \alpha \operatorname{Tr}_{L(\sqrt{\delta}) / L}\left(u_{L}\right),-\delta\right)$ over $L$ is split.

Let $v$ be an ordering on $L$ which extends to an ordering $w$ on $L(\sqrt{\delta})$. Then $\delta>{ }_{v} 0$ and hence $2 \alpha \operatorname{Tr}_{L(\sqrt{\delta}) / L}\left(u_{L}\right)>{ }_{v} 0$. Let bar denote the non-trivial automorphism of $L(\sqrt{\delta})$ over $L$. Since $\alpha u_{L} \overline{\alpha u_{L}}=\alpha^{2}>_{v} 0$, both $\alpha u_{L}$ and $\overline{\alpha u_{L}}$ have the same sign at $w$. But $\alpha \operatorname{Tr}_{L(\sqrt{\delta}) / L}\left(u_{L}\right)=\alpha\left(u_{L}+\overline{u_{L}}\right)>_{v} 0$. Thus $\alpha u_{L}>_{w} 0$. This is true for every ordering of $L(\sqrt{\delta})$. Thus $\alpha u_{L} \in \Sigma(L(\sqrt{\delta}))$ and $N_{L(\sqrt{\delta}) / L}\left(u_{L}\right)=1$. This completes the proof of the lemma.

Let $D$ be a division algebra with centre $Z$ and let $\tau$ be an involution on $D$ of the second kind. Let $Z^{\tau}=F$. Let $(V, h)$ be a non-degenerate hermitian space over $(D, \tau)$. Then the integer $\operatorname{dim}_{D}(V)$ is said to be the rank of $h$ and is denoted by $\operatorname{rank}(h)$. Let $\operatorname{rank}(h)=n$. For a choice $\left\{e_{1}, e_{2}, \cdots, e_{n}\right\}$ of a $D$-basis of $V$, the form $h$ determines a matrix $M_{h}=\left(h\left(e_{i}, e_{j}\right)\right) \in M_{n}(D)$. The matrix $M_{h}$ is $\tau$-hermitian symmetric. Let $r=n \operatorname{deg}(D)$. We define the discriminant of $h$ to be $(-1)^{r(r-1) / 2} \operatorname{Nrd}\left(M_{h}\right) \in F^{*} / N_{Z / F}\left(F^{*}\right)$ and denote it by $\operatorname{disc}(h)$.

We refine the notion of discriminant to the notion of Discriminant as follows:

Let $M_{h} \in M_{n}(D)$ be a matrix as above, representing the hermitian form $h$. Let $M_{h}^{\prime} \in M_{n}(D)$ also represent $h$. Then there exists an invertible matrix $T \in M_{n}(D)$ such that

$$
\operatorname{Nrd}\left(M_{h}^{\prime}\right)=\operatorname{Nrd}\left(M_{h}\right) \operatorname{Nrd}(T) \tau(\operatorname{Nrd}(T)) .
$$

Thus we have the following well defined notion of Discriminant:

$$
\operatorname{Disc}(h)=(-1)^{r(r-1) / 2} \operatorname{Nrd}\left(M_{h}\right) \in F^{*} / N_{Z / F}\left(\operatorname{Nrd}\left(D^{*}\right)\right)
$$

where $r=n \operatorname{deg}(D)$.

We now quote a classification result for hermitian forms over division algebras with an involution of the second kind over fields with $\operatorname{vcd}(F) \leq 2$.

Theorem 5.8 ([BP2, Theorem 4.8]). Let $F$ be a field with $\operatorname{vcd}(F) \leq 2$ and let $D$ be a division algebra with an involution $\tau$ of the second kind such that $(\operatorname{centre}(D))^{\tau}=$ $F$. Let $h$ be a hermitian form over $(D, \tau)$, Then $h$ is hyperbolic if and only if $\operatorname{rank}(h)$ is even, $\operatorname{Disc}(h)$ is trivial and $h$ has trivial signature.

Lemma 5.9. Let $D$ be a central division algebra over $Z$, $\tau$ be a $Z / F$-involution over $D$ and $h$ be a hermitian of rank $2 s$ over $(D, \tau)$. Let $\operatorname{disc}(h)=1$. Then

$$
F^{*}=\left\langle\left\{N_{M / F}\left(M^{*}\right): \operatorname{Disc}\left(h_{M}\right)=1\right\}\right\rangle \text {. }
$$


Proof. Let $M_{h} \in M_{2 s}(D)$ be a matrix representing $h$. Since $\operatorname{disc}(h)=1 \epsilon$ $F^{*} / N_{Z / F}\left(Z^{*}\right)$, we have $\operatorname{Nrd}\left(M_{h}\right)=d \in N_{Z / F}\left(Z^{*}\right)$. Let $z \in Z$ be such that $d=N_{Z / F}(z)$. Let $\beta=\operatorname{Tr}_{Z / F}(z)$ and $\gamma=z \beta^{-1}$. Let $w$ be an ordering on $Z$ which extends an ordering $v$ of $F$ such that $D_{w}$ is not split. $\operatorname{Then} \operatorname{Nrd}\left(M_{h}\right)=$ $d=N_{Z / F}(z)>_{w} 0$. Thus $\operatorname{Tr}_{Z / F}(z)=\beta>_{w} 0$ if and only if $z>_{w} 0$. This implies that $\gamma=z \beta^{-1}>_{w} 0$ and thus by 1.8, $\gamma \in \operatorname{Nrd}\left(D^{*}\right)$. Let $x \in D^{*}$ be such that $\operatorname{Nrd}(x)=\gamma$. Let

$$
M_{h}^{\prime}=\left(\begin{array}{cccc}
1 & & & \\
& 1 & & \\
& & . & \\
& & & x
\end{array}\right) M_{h}\left(\begin{array}{cccc}
1 & & & \\
& 1 & & \\
& & . & \\
& & & \tau(x)
\end{array}\right)^{t} .
$$

Then $\operatorname{Nrd}\left(M_{h}^{\prime}\right)=\left(d \beta^{-1}\right)^{2}$ and we conclude that for a suitable choice of a matrix $M_{h}$ representing the hermitian form $h, \operatorname{Nrd}\left(M_{h}\right)=\alpha^{2}, \alpha \in F^{*}$. Let $\lambda \in F^{*}$. By 5.7, there exist quadratic extensions $L_{i} / F, \lambda_{i} \in L_{i}^{*}$ and $u_{i} \in L_{i}(\sqrt{\delta}), 1 \leq i \leq r$, such that $\lambda=\prod_{i} N_{L_{i} / F}\left(\lambda_{i}\right), \alpha u_{i} \in \Sigma(L(\sqrt{\delta}))$ and $N_{L_{i}(\sqrt{\delta}) / L}\left(u_{i}\right)=1$. Then

$$
\alpha^{2}=N_{L_{i}(\sqrt{\delta}) / L_{i}}\left(\alpha u_{i}\right) \in N_{L_{i}(\sqrt{\delta}) / L_{i}}\left(\operatorname{Nrd}\left(D_{L_{i}(\sqrt{\delta})}\right)\right)
$$

and hence $\operatorname{Disc}\left(h_{L_{i}}\right)=1$ for $1 \leq i \leq r$. Thus $\lambda \in\left\langle\left\{N_{M / F}\left(M^{*}\right): \operatorname{Disc}\left(h_{M}\right)=1\right\}\right\rangle$ and we conclude that $F^{*}=\left\langle\left\{N_{M / F}\left(M^{*}\right): \operatorname{Disc}\left(h_{M}\right)=1\right\}\right\rangle$.

The following propositions are used in the proof of 5.13, which is the main result of this section.

Proposition 5.10. Let $A \simeq M_{r}(D)$ where $D$ is a division algebra over $Z$ and $r$ is even. Let $\sigma$ be a locally hyperbolic $Z / F$-involution on $A$. Then $\operatorname{Hyp}(A, \sigma)=F^{*}$.

Proof. Let $\sigma$ be adjoint to a hermitian form $h$ of rank $r$. Let $d \in F^{*} / N_{Z / F}\left(Z^{*}\right)$ denote the discriminant of $h$. Since $\sigma$ is locally hyperbolic, for each $v \in \Omega$, the quaternion algebra $(\delta, d)$ splits at $F_{v}$. Thus by $1.8, \operatorname{Nrd}((\delta, d))=F^{*}$. Let $\lambda \in F^{*}$. There exists a finite extension $E / F$ such that $\lambda \in N_{E / F}\left(E^{*}\right)$ and $(\delta, d)$ splits over $E$. Then $\operatorname{disc}\left(h_{E}\right)$ is trivial. By 5.9 we have

$$
E^{*}=\left\langle\left\{N_{M / E}\left(M^{*}\right): \operatorname{Disc}\left(h_{M}\right)=1\right\} .\right.
$$

Let $M / E$ be an extension such that $\operatorname{Disc}\left(h_{M}\right)=1$. Since $\sigma$ is locally hyperbolic, $\operatorname{sgn}\left(h_{M}\right)=0$. Thus by 5.8 , the form $h_{M}$ is hyperbolic and $\operatorname{Hyp}\left(h_{M}\right)=M^{*}$. Hence by $(*), \operatorname{Hyp}\left(h_{E}\right)=E^{*}$ and

$$
\lambda \in N_{E / F}\left(E^{*}\right)=N_{E / F}\left(\operatorname{Hyp}\left(h_{E}\right)\right) \subseteq \operatorname{Hyp}(h)=\operatorname{Hyp}(A, \sigma)
$$

which implies that $\operatorname{Hyp}(A, \sigma)=F^{*}$. This completes the proof.

Proposition 5.11. Let $A$ be a central simple algebra over $Z$ with $\operatorname{deg}(A) \equiv 0(4)$. Let $\exp (A)=2^{m}$ for some positive integer $m$. Let $\sigma$ be a locally hyperbolic $Z / F$ involution on $A$. Then $\operatorname{Hyp}(A, \sigma)=F^{*}$.

Proof. Suppose $m=1$. Since $\operatorname{cores}_{Z / F}(A)=0$, by $1.12 A \sim A_{0} \otimes_{F} Z$ for some central simple $F$-algebra $A_{0}$ with $\exp \left(A_{0}\right)=2$. Let $M$ be a finite extension of $F$ such that $A_{0 M} \sim H$ for some quaternion algebra $H$ over $M$. Then $A_{M}=M_{r}\left(H \otimes_{F} Z\right)$. 
Since $\operatorname{deg}(A) \equiv 0(4)$, the integer $r$ is even. Thus by $5.10, \operatorname{Hyp}\left(A_{M}, \sigma_{M}\right)=M^{*}$. In view of 2.4 we have

$$
F^{*}=\left\langle\left\{N_{M / F}\left(M^{*}\right): A_{0 M} \sim H \text { for some quaternion algebra } H \text { over } M\right\}\right\rangle .
$$

It follows that $\operatorname{Hyp}(A, \sigma)=F^{*}$.

Suppose $m \geq 2$. Since $\operatorname{Br}\left(Z_{w}\right)=\mathbb{Z} / 2 \mathbb{Z}$ for each ordering $w \in \Omega_{Z}$, the algebra $A \otimes_{Z} A$ splits locally. Clearly $\exp \left(A \otimes_{Z} A\right)=2^{m-1}$ and $\operatorname{cores}_{Z / F}\left(A \otimes_{Z} A\right)=0$. Let $\lambda \in F^{*}$. By 2.6, there exist extensions $L_{i} / F, 1 \leq i \leq s$, and $\lambda_{i} \in L_{i}^{*}$ such that each $\left(A \otimes_{Z} A\right) \otimes_{F} L_{i}$ is split and $\lambda=\prod_{i} N_{L_{i} / F}\left(\lambda_{i}\right)$. Then $\exp \left(A \otimes_{F} L_{i}\right)=2$ for each $i$ and by the case $m=1, \lambda_{i} \in \operatorname{Hyp}\left(A_{L_{i}}, \sigma_{L_{i}}\right)$. Hence

$$
\lambda=\prod_{i} N_{L_{i} / F}\left(\lambda_{i}\right) \in \prod_{i} N_{L_{i} / F}\left(\operatorname{Hyp}\left(A_{L_{i}}, \sigma_{L_{i}}\right)\right) \subseteq \operatorname{Hyp}(A, \sigma),
$$

and it follows that $\operatorname{Hyp}(A, \sigma)=F^{*}$.

Proposition 5.12. Let $A$ be a central simple algebra over $Z$ with $\operatorname{deg}(A) \equiv 0(4)$. Let $\sigma$ be a locally hyperbolic $Z / F$-involution on $A$. Then we have $\operatorname{Hyp}(A, \sigma) \cdot F^{* 2}=$ $F^{*}$.

Proof. By [BP1, Lemma 3.3.1], there exists an odd degree extension $M$ of $F$ such that $\exp \left(A_{M}\right)$ is a power of 2 and by $5.11, \operatorname{Hyp}\left(A_{M}, \sigma_{M}\right)=M^{*}$. Taking norm from $M / F$ and using that $[M: F]$ is odd, we conclude that $\operatorname{Hyp}(A, \sigma) \cdot F^{* 2}=F^{*}$.

Theorem 5.13. Let $F$ be a field with $\operatorname{vcd}(F) \leq 2$ and let $Z$ be a quadratic extension over $F$. Let $A$ be a central simple algebra over $Z$ and let $\sigma$ be a $Z / F$-involution on A. Then $G(A, \sigma) \subseteq \operatorname{Hyp}(A, \sigma) \cdot N_{Z / F}\left(Z^{*}\right)$.

Proof. The cases where $\operatorname{deg}(A)$ is odd or $\operatorname{deg}(A) \equiv 2(4)$ are covered by 1.6 and 5.6 respectively. We assume that $\operatorname{deg}(A) \equiv 0(4)$. Let $\lambda \in G(A, \sigma)$. At each $v \in \Omega$, the involution $\sigma_{v}$ is adjoint to an even rank hermitian form which is hyperbolic if and only if $\operatorname{sgn}\left(\sigma_{v}\right)=0$. Therefore $\lambda>_{v} 0$ at those $v \in \Omega$, where $\sigma_{v}$ is not hyperbolic. Let $K=F(\sqrt{-\lambda})$. Then $\operatorname{deg}\left(A_{K}\right) \equiv 0(4)$ and $\sigma_{K}$ is locally hyperbolic. Thus by 5.12 , we have $\operatorname{Hyp}\left(A_{K}, \sigma_{K}\right) \cdot K^{* 2}=K^{*}$. Let $\sqrt{-\lambda}=\alpha \beta^{2}$, where $\alpha \in \operatorname{Hyp}\left(A_{K}, \sigma_{K}\right)$ and $\beta \in K^{*}$. Then $\lambda=N_{K / F}(\sqrt{-\lambda})=N_{K / F}(\alpha)\left(N_{K / F}(\beta)\right)^{2}$. But $N_{K / F}(\alpha) \in$ $N_{K / F}\left(\operatorname{Hyp}\left(A_{K}, \sigma_{K}\right)\right) \subseteq \operatorname{Hyp}(A, \sigma)$. Thus $\lambda \in \operatorname{Hyp}(A, \sigma) . F^{* 2}$. This completes the proof.

\section{Fields with $\operatorname{vcd}(F) \leq 2$ : Orthogonal Groups}

Let $F$ be an arbitrary field with $\operatorname{char}(F) \neq 2$. Let $D$ be a central division algebra over $F$ with an orthogonal involution $\tau$. We first recall from [BP2], certain invariants associated to hermitian forms over $(D, \tau)$.

Discriminant: Let $D$ and $\tau$ be as above and let $h$ be a hermitian form of even rank over $(D, \tau)$. Let $\operatorname{rank}(h)=2 m$ and let $M_{h} \in M_{2 m}(D)$ represent the hermitian form $h$. Let

$$
\operatorname{Disc}(h)=(-1)^{r(r-1) / 2} \operatorname{Nrd}\left(M_{h}\right) \in F^{*} /\left(\operatorname{Nrd}\left(D^{*}\right)\right)^{2},
$$

where $r=2 m \operatorname{deg}(D)$. If $M_{h}^{\prime} \in M_{2 m}(D)$ is another matrix representing $h$, then there exists an invertible matrix $T \in M_{2 m}(D)$ such that $M_{h}=T M_{h}\left(\tau(T)^{t}\right)$. Thus $\operatorname{Nrd}\left(M_{h}^{\prime}\right)=\operatorname{Nrd}\left(M_{h}\right) \operatorname{Nrd}(T)^{2}$ and $\operatorname{Disc}(h)$ is well defined. We call $\operatorname{Disc}(h)$ the Discriminant of $h$. 
Clifford invariant: We recall from [KMRT, $\S 8 . \mathrm{B}]$, the notion of the Clifford algebra $\mathrm{C}(A, \sigma)$ associated to a central simple algebra $A$ over a field $F$ with an involution $\sigma$ of orthogonal type. If $A$ is split and $\sigma$ is adjoint to a quadratic form $q$, then $\mathrm{C}(A, \sigma)$ is the even Clifford algebra $\mathrm{C}_{0}(q)$ of the quadratic form $q$. If $\operatorname{disc}(\sigma)$ is trivial, $\mathrm{C}(A, \sigma)$ decomposes into a product $\mathrm{C}_{+}(A, \sigma) \times \mathrm{C}_{-}(A, \sigma)$, each of the factors being a central simple algebra over $F$ such that

$$
\left[\mathrm{C}_{+}(A, \sigma)\right]+\left[\mathrm{C}_{-}(A, \sigma)\right]=[A] \in \operatorname{Br}(F) .
$$

Let $D, \tau$ and $h$ be as above. Let $\operatorname{disc}(h)$ be trivial and $A=M_{2 m}(D)$. Let $\tau_{h}$ be the orthogonal involution on $A$ which is adjoint to $h$. We define the Clifford invariant of $h$ as follows:

$$
\mathcal{C} \ell(h)=\left[C_{+}\left(M_{2 m}(D), \tau_{h}\right)\right] \in \operatorname{Br}(F) /[D] .
$$

Let $H_{2 m}$ denote the matrix $\left(\begin{array}{cc}0 & I_{m} \\ I_{m} & 0\end{array}\right) \in M_{2 m}(D)$ where $I_{m}$ is the identity matrix of size $m$. The matrix $H_{2 m}$ represents the hyperbolic form of rank $2 m$ over $(D, \tau)$. Let $\mathrm{U}_{2 m}(D, \tau), \mathrm{SU}_{2 m}(D, \tau)$ and $\operatorname{Spin}_{2 m}(D, \tau)$ denote respectively, the unitary, special unitary group and spin group with respect to the hyperbolic form $H_{2 m}$ over $(D, \tau)$. We have an exact sequence

$$
1 \rightarrow \mu_{2} \rightarrow \operatorname{Spin}_{2 m}(D, \tau) \rightarrow \mathrm{SU}_{2 m}(D, \tau) \rightarrow 1
$$

from which one gets the exact sequence of pointed sets

$$
\rightarrow H^{1}\left(F, \operatorname{Spin}_{2 m}(D, \tau)\right) \rightarrow H^{1}\left(F, \mathrm{SU}_{2 m}(D, \tau)\right) \stackrel{\delta}{\rightarrow} H^{2}\left(F, \mu_{2}\right) .
$$

Let $\mathfrak{S}$ denote the set of ordered pairs $(X, a)$, where $X \in \mathrm{GL}_{2 m}(D)$ and $a \in F^{*}$ satisfy $\tau(X)=X^{t}$ and $\operatorname{Nrd}(X)=\operatorname{Nrd}\left(H_{2 m}\right) a^{2}$. The elements of $H^{1}\left(F, \mathrm{SU}_{2 m}(D, \tau)\right)$ are equivalence classes of $\mathfrak{S}$ under the following equivalence relation: $(X, a) \sim$ $\left(X^{\prime}, a^{\prime}\right)$ if and only if there exists $Y \in \mathrm{GL}_{2 m}(D)$ with $X^{\prime}=Y X \bar{Y}^{t}$ and $a^{\prime}=$ $\operatorname{Nrd}(Y) a$.

Let $h$ be a hermitian form over $(D, \tau)$ with $\operatorname{rank}(h)=2 m$ and $\operatorname{disc}(h)=1$. Let $M_{h}$ be a matrix which represents $h$ and $\operatorname{Nrd}\left(M_{h}\right)=a^{2}, a \in F^{*}$. The two elements $\xi_{a}=\left(M_{h}, a\right)$ and $\xi_{-a}=\left(M_{h},-a\right)$ in $H^{1}\left(F, \mathrm{SU}_{2 m}(D, \tau)\right)$ map to $[h]$ under $H^{1}\left(F, \mathrm{SU}_{2 m}(D, \tau)\right) \rightarrow H^{1}\left(F, \mathrm{U}_{2 m}(D, \tau)\right)$. Let $C_{+}(h)=\delta\left(\xi_{a}\right)$ and $C_{-}(h)=\delta\left(\xi_{-a}\right)$. We recall the following lemma from [BMPS, Lemma 3.1].

Lemma 6.1. If $F$ is a formally real field and $v$ is an ordering on $F$ such that $D_{v}$ is not split, then the algebra $C_{+}(h)$ is split at $v$ if and only if $a>_{v} 0$.

Rost invariant: Let $h$ be a hermitian form over $(D, \tau)$ with $\operatorname{rank}(h)=2 m$, trivial discriminant and trivial Clifford invariant. Consider the exact sequence

$$
1 \rightarrow \mathrm{SU}_{2 m}(D, \tau) \rightarrow \mathrm{U}_{2 m}(D, \tau) \rightarrow \mu_{2} \rightarrow 1
$$

This gives rise to the following exact sequence of pointed sets:

$$
\rightarrow \mathrm{U}_{2 m}(D, \tau)(F) \rightarrow\{ \pm 1\} \rightarrow H^{1}\left(F, \mathrm{SU}_{2 m}(D, \tau)\right) \rightarrow H^{1}\left(F, \mathrm{U}_{2 m}(D, \tau)\right) \rightarrow .
$$

Since $\mathcal{C} \ell(h)=0$, there exists $\xi \in H^{1}\left(F, \mathrm{SU}_{2 m}(D, \tau)\right)$ which maps to the class of $h$ in $H^{1}\left(F, \mathrm{U}_{2 m}(D, \tau)\right)$ such that $\delta(\xi)=0$. Let $\tilde{\xi} \in H^{1}\left(F, \operatorname{Spin}_{2 m}(D, \tau)\right)$ be a preimage of $\xi \in H^{1}\left(F, \operatorname{SU}_{2 m}(D, \tau)\right)$. Let $G=\operatorname{Spin}_{2 m}(D, \tau)$ and $\mathcal{R}_{G}: H^{1}(F, G) \rightarrow$ 
$H^{3}(F, \mathbb{Q} / \mathbb{Z}(2))$ denote the Rost invariant of $G[\mathrm{Me} 3]$. The Rost invariant of $h$ is defined as follows ([BP2, pp. 664]):

$$
R(h)=\mathcal{R}_{G}(\tilde{\xi}) \in \frac{H^{3}(F, \mathbb{Q} / \mathbb{Z}(2))}{F^{*} \cup[D]} .
$$

The element $\mathcal{R}_{G}(\tilde{\xi})$ takes values in $H^{3}(F, \mathbb{Z} / 4)$ [BP2, Remark 1 ], where $\mathbb{Z} / 4$ has the trivial Galois module structure. We now recall a proposition which we shall use often.

Proposition 6.2 ([BP2, Cor. 2.6]). Let $F$ be a formally real field and let $I^{3}(F)$ be torsion-free. Let $\Omega$ be the set of orderings on $F$. Then the natural map

$$
H^{3}(F, \mathbb{Z} / 4) \rightarrow \prod_{v \in \Omega} H^{3}\left(F_{v}, \mathbb{Z} / 4\right)
$$

is injective.

We now record a classification result for hermitian forms over central division algebras with orthogonal involutions over fields with $\operatorname{vcd}(F) \leq 2$.

Theorem 6.3 ([BP2, Th. 7.3]). Let $F$ be a field with $\operatorname{vcd}(F) \leq 2$ and let $D$ be a central division algebra over $D$ with an orthogonal involution $\tau$. Let $h$ be a hermitian form over $(D, \tau)$. Then $h$ is hyperbolic if and only if $h$ has even rank, trivial Discriminant, trivial Clifford and Rost invariant and trivial signature.

Let $F$ be a field with $\operatorname{vcd}(F) \leq 2$ and let $(A, \sigma)$ be a central simple algebra over $F$ with orthogonal involution. If $A$ is split, $\operatorname{deg}(A)$ is even, $\sigma$ is locally hyperbolic and $\operatorname{disc}(\sigma)=1$, so that by 5.2 we have $\operatorname{Hyp}(A, \sigma)=F^{*}$. We now consider the case where $A$ is locally split.

Lemma 6.4. Let $\operatorname{vcd}(F) \leq 2$ and let $A$ be a central simple algebra of even degree over $F$. Let $\sigma$ be an orthogonal involution on $A$. If $A$ is locally split, then

$$
G_{+}(A, \sigma)=\operatorname{Hyp}(A, \sigma) \cdot F^{* 2} \text {. }
$$

Proof. Let $\lambda \in G_{+}(A, \sigma)$ and $K=F(\sqrt{-\lambda})$. Clearly $\lambda \in N_{K / F}\left(K^{*}\right)$. Let $\operatorname{disc}(\sigma)=$ $d$ and $L=F(\sqrt{d})$. By 1.3, $\lambda \in N_{L / F}\left(L^{*}\right)$. Let $M=F(\sqrt{-\lambda}, \sqrt{d})$. Using [W, Lemma 2.14] for the biquadratic extension $M / F$, there exist $x \in M^{*}$ and $y \in F^{*}$ such that $\lambda=N_{M / F}(x) y^{2}$. Further $A_{M}$ is locally split and by $1.8, \operatorname{Nrd}\left(A_{M}\right)=M^{*}$. Let $E / M$ be an extension such that $x=N_{E / M}(\alpha)$ for some $\alpha \in E^{*}$, and let $A_{E}$ be split. Clearly $\operatorname{disc}\left(\sigma_{E}\right)=1, \sigma_{E}$ is locally hyperbolic and $A_{E}$ is split. Thus by $5.2, \operatorname{Hyp}\left(A_{E}, \sigma_{E}\right)=E^{*}$ and hence $x=N_{E / M}(\alpha) \in \operatorname{Hyp}\left(A_{M}, \sigma_{M}\right)$. Thus $\lambda=N_{M / F}(x) y^{2} \subseteq \operatorname{Hyp}(A, \sigma) \cdot F^{* 2}$. We conclude that $G_{+}(A, \sigma) \subseteq \operatorname{Hyp}(A, \sigma) . F^{* 2}$. In view of 1.1 we have $G_{+}(A, \sigma)=\operatorname{Hyp}(A, \sigma) \cdot F^{* 2}$.

We continue with some lemmas which will be used in the proofs of the main results of this section.

Lemma 6.5. Let $\operatorname{vcd}(F) \leq 2$ and $\chi \in H^{3}\left(F, \mu_{2}\right)$. Then

$$
F^{*}=\left\langle\left\{N_{L / F}\left(L^{*}\right): L \in \mathcal{F}_{2}(F) \text { and } \chi_{L}=(-1) \cup(-1) \cup(-x) \text { for some } x \in L^{*}\right\}\right\rangle \text {. }
$$

Proof. Since $\operatorname{vcd}(F) \leq 2, H^{3}\left(F(\sqrt{-1}), \mu_{2}\right)=0$ and in view of the Arason exact sequence 1.11 , the map $H^{2}\left(F, \mu_{2}\right) \stackrel{\cup(-1)}{\longrightarrow} H^{3}\left(F, \mu_{2}\right)$ is surjective. Let $\xi \in H^{2}\left(F, \mu_{2}\right)$ be such that $(-1) \cup \xi=\chi$. Let $D_{\xi}$ be a central division algebra over $F$, whose 
Brauer class is represented by $\xi$. Then $\exp \left(D_{\xi}\right)=2$. Let $L \in \mathcal{F}_{2}(F)$ be such that $\left(D_{\xi}\right)_{L} \sim(-1) \cup(-x)$ for some $x \in L$. Then

$$
\chi_{L}=(-1) \cup \xi_{L}=(-1) \cup\left(D_{\xi}\right)_{L}=(-1) \cup(-1) \cup(-x) .
$$

In view of this and 2.4, we have

$$
\begin{aligned}
F^{*} & =\left\langle\left\{N_{L / F}\left(L^{*}\right): L \in \mathcal{F}_{2}(F) \text { and }\left(D_{\xi}\right)_{L}=(-1) \cup(-x) \text { for some } x \in L^{*}\right\}\right\rangle \\
& \subseteq\left\langle\left\{N_{L / F}\left(L^{*}\right): L \in \mathcal{F}_{2}(F) \text { and } \chi_{L}=(-1) \cup(-1) \cup(-x) \text { for some } x \in L^{*}\right\}\right\rangle .
\end{aligned}
$$

For $\chi \in H^{r}\left(F, \mu_{2}\right)$, we set $N(\chi)=\left\langle\left\{N_{L / F}\left(L^{*}\right): \chi_{L}=0\right\}\right\rangle$.

Lemma 6.6. Let $\operatorname{vcd}(F) \leq 2$ and $\chi \in H^{r}\left(F, \mu_{2}\right), r \geq 2$. Then the following three groups coincide:

(i) $N(\chi)$.

(ii) $\left\{\lambda \in F^{*}: \lambda>_{v} 0\right.$ at those $v \in \Omega$ where $\left.\chi_{v} \neq 0\right\}$.

(iii) $\left\{\lambda \in F^{*}:(\lambda) \cup \chi=0\right\}$.

Proof. Since $\operatorname{vcd}(F) \leq 2$, in view of 1.15 the cohomology groups $H^{r+1}\left(F, \mu_{2}\right)$ are (-1)-torsion-free for $r \geq 2$ and thus the groups (ii) and (iii) coincide. We show that $N(\chi) \subseteq\left\{\lambda \in F^{*}:(\lambda) \cup \chi=0\right\}$. Let $\lambda \in N(\chi)$ be such that $\lambda=N_{L / F}(\mu)$ for an extension $L / F$ with $\chi_{L}=0$. Then $((\mu) \cup \chi)_{L}=0$ and thus we have

$$
\operatorname{cores}_{L / F}((\mu) \cup \chi)_{L}=(\lambda) \cup \chi=0 .
$$

Hence $N(\chi) \subseteq\left\{\lambda \in F^{*}:(\lambda) \cup \chi=0\right\}$. To complete the proof, we show that $\left\{\lambda \in F^{*}: \lambda>_{v} 0\right.$ at those $v \in \Omega$ where $\left.\chi_{v} \neq 0\right\} \subseteq N(\chi)$. Let $\lambda \in F^{*}$ be such that $\lambda>_{v} 0$ at those $v \in \Omega$ where $\chi_{v} \neq 0$. Let $L=F(\sqrt{-\lambda})$. Then it follows that $\chi_{w}=0$ for each ordering $w$ of $L$. It follows from [Ar, Th. 2.1] that $\operatorname{ved}(L) \leq 2$ and thus by $1.15, H^{3}\left(L, \mu_{2}\right)$ is $(-1)$-torsion-free. Therefore $\chi_{L}=0$. Thus $\lambda=$ $N_{L / F}(\sqrt{-\lambda}) \in N(\chi)$. This completes the proof.

In $6.7,6.8$ and 6.9 below, the only restriction on $F$ is that $\operatorname{char}(F) \neq 2$. Let $D$ be a central division algebra over $F$ and let $\tau$ be an orthogonal involution on $D$. Let $h$ be a hermitian form of rank $2 m$ and trivial discriminant over $(D, \tau)$. Let $a \in F^{*}$ be such that $\operatorname{Nrd}\left(M_{h}\right)=a^{2}$, where $M_{h}$ is a matrix representing the form $h$. Since $\operatorname{disc}(h)=1$, we recall from [MT, Prop. 1.12] that $G_{+}(h)=G(h)$.

Lemma 6.7. Let $D$ be a central division algebra over a field $F$ of characteristic different from 2 with an orthogonal involution $\tau$. Let $h$ and $h^{\prime}$ be two even rank hermitian forms of trivial discriminant over $(D, \tau)$. Then we have the following additive property for Clifford invariants:

$$
\mathcal{C} \ell\left(h \perp h^{\prime}\right)=\mathcal{C} \ell(h)+\mathcal{C} \ell\left(h^{\prime}\right) \in H^{2}\left(F, \mu_{2}\right) /[D] .
$$

Proof. We extend the scalars to the function field of the Brauer-Severi variety of $D$. Using the fact that the invariant $e_{2}$ of quadratic forms is additive on forms of trivial discriminant and that the kernel of the scalar extension map $H^{2}\left(F, \mu_{2}\right) \rightarrow$ $H^{2}\left(F\left(X_{D}\right), \mu_{2}\right)$ is generated by the class of $D$ in $H^{2}\left(F, \mu_{2}\right)$ [MT, Cor. 2.7], the lemma follows. 
From this lemma and the fact that two similar hermitian forms with even rank and trivial discriminant have the same Clifford invariants [BP1, pp. 204], we immediately have

Corollary 6.8. Let $D, \tau$ and $h$ be as in 6.7. Then for each $\lambda \in F^{*}$, the Clifford invariant $\mathcal{C} \ell(h \perp-\lambda h)$ is trivial.

In the following lemma, we compute the Rost invariant of the hermitian form $h \perp-\lambda h$, where $h$ is as in 6.7 and $\lambda \in F^{*}$ is an arbitrary scalar.

Lemma 6.9. Let $D$ be a central division algebra of even degree over a field $F$ of characteristic different from 2. Let $\tau$ be an orthogonal involution on $D$ and let $h$ be a hermitian form over $(D, \tau)$ of even rank and trivial discriminant. Let $\lambda \in F^{*}$. Then,

$$
R(h \perp-\lambda h)=(\lambda) \cup\left[C_{+}(h)\right] \in H^{3}(F, \mathbb{Q} / \mathbb{Z}(2)) / F^{*} \cup[D] .
$$

Proof. Let $\operatorname{rank}(h)=2 m$ and $A=M_{2 m}(D)$. Let $\tau_{h}$ be the involution on $A$ which is adjoint to $h$. We denote by $\mathbf{P G O}(h)$ the group $\mathbf{P S i m}_{+}\left(A, \tau_{h}\right)$ of similitudes. We have an exact sequence

$$
1 \rightarrow \mu_{2} \rightarrow \mathrm{SU}(h) \rightarrow \mathbf{P G O}_{+}(h) \rightarrow 1
$$

which induces a map on the cohomology sets $H^{1}\left(F, \mu_{2}\right) \rightarrow H^{1}(F, \mathrm{SU}(h))$. We claim that under this map $(\lambda) \in H^{1}\left(F, \mu_{2}\right)$ is mapped to an element $\xi_{\lambda} \in H^{1}(F, \mathrm{SU}(h))$ which corresponds to the class of the hermitian form $\lambda h$ in $H^{1}(F, \mathrm{U}(h))$. In fact, the cocycle $(\lambda) \in Z^{1}\left(F, \mu_{2}\right)$ given by $s \mapsto s(\sqrt{\lambda})(\sqrt{\lambda})^{-1}$ for $s \in \operatorname{Gal}\left(F_{s} / F\right)$, when treated as a cocycle with values in $\mathrm{U}(h)$, represents $[\lambda h]$ in $H^{1}(F, \mathrm{U}(h))$.

Since $\operatorname{deg}(A) \equiv 0(4)$, the centre of $\operatorname{Spin}(h)$ is $\mu_{2} \times \mu_{2}$ and the kernel of the map $\operatorname{Spin}(h) \rightarrow \operatorname{SU}(h)$ is $(\epsilon, \epsilon)$, where $\epsilon= \pm 1$. The quotient of $\mu_{2} \times \mu_{2}$ by $\mu_{2}$ under the diagonal embedding maps isomorphically onto the centre of $\mathrm{SU}(h)$. By [MPT, Th. 1.14], the Rost invariant of the image $\tilde{\xi}_{\lambda}$ of $(1, \lambda) \in H^{1}\left(F, \mu_{2} \times \mu_{2}\right)$ in $H^{1}(F$, $\operatorname{Spin}(h))$ is $(\lambda) \cup\left[C_{+}(h)\right]$. Thus $\tilde{\xi}_{\lambda} \in H^{1}(F, \operatorname{Spin}(h))$ maps to $\xi_{\lambda} \in H^{1}(F, \operatorname{SU}(h))$, which in turn maps to the class of $\lambda h$ in $H^{1}(F, \mathrm{U}(h))$ as is seen above. Thus we conclude that the hermitian form $\lambda h$ admits a lift $\tilde{\xi}_{\lambda}$ such that $\mathcal{R}\left(\tilde{\xi}_{\lambda}\right)=(\lambda) \cup\left[C_{+}(h)\right]$.

We now compute $R(h \perp-\lambda h)$. Let $i: \operatorname{Spin}(-h) \rightarrow \operatorname{Spin}(-h \perp h)$ be the natural map and $\tilde{i}: H^{1}(F, \operatorname{Spin}(-h)) \rightarrow H^{1}(F, \operatorname{Spin}(-h \perp h))$ the induced map on the cohomology sets. In view of [BP2, Lemma 3.6], $\mathcal{R}(\tilde{i}(\xi))=\mathcal{R}(\xi)$ for every $\xi \in$ $H^{1}(F, \operatorname{Spin}(-h))$. The group $\operatorname{Spin}(-h \perp h)$ maps isomorphically onto $\operatorname{Spin}_{4 m}(D, \tau)$ preserving the Rost invariant. Further, the image of $(1, \lambda)$ in the cohomology set $H^{1}(F, \operatorname{Spin}(-h))$ maps to the isometry class of $-\lambda h$ in $H^{1}(F, \mathrm{U}(h))$ and to the isometry class of $-\lambda h \perp h$ in $H^{1}\left(F, \mathrm{U}_{4 n}(D, \tau)\right)$. This implies that the Rost invariant $R(h \perp-\lambda h)$ is equal to $(\lambda) \cup\left[C_{+}(h)\right] \in H^{3}(F, \mathbb{Q} / \mathbb{Z}(2)) / F^{*} \cup[D]$. This completes the proof.

From now on, we assume that $\operatorname{vcd}(F) \leq 2$. Let $L / F$ be a formally real extension and let $\Omega_{L}$ be the set of orderings on $L$. Let $h$ be a hermitian form over $(D, \tau)$ - a central division algebra $D$ over $F$ with an orthogonal involution $\tau$. We define $\mathcal{S}_{\ell, L}(h)$ as follows:

$$
\mathcal{S}_{\ell, L}(h)=\left\{\lambda \in L^{*}: h_{L_{w}} \simeq \lambda h_{L_{w}} \text { for all } w \in \Omega_{L}\right\} .
$$

If $L=F$, then we simply write $\mathcal{S}_{\ell}(h)$ to denote $\mathcal{S}_{\ell, F}(h)$. In $6.10-6.14$ below, $h$ denotes an even rank hermitian form over $(D, \tau)$ with trivial discriminant 
and $\operatorname{rank}(h) \cdot \operatorname{degree} D \equiv 0(4)$. Further, $a \in F^{*}$ denotes a scalar which satisfies $\operatorname{Nrd}\left(M_{h}\right)=a^{2}$ for a choice $M_{h}$ of a matrix representing $h$. Further, for $z \in F^{*}$ and a central simple algebra $B$ over $F$ with $\exp (B)=2$, we denote by $z \cup B$ the element $(z) \cup[B] \in H^{3}\left(F, \mu_{2}\right)$.

Proposition 6.10. We have $G(h)=(N(a \cup D) N(-a \cup D)) \cap \mathcal{S}_{\ell}(h)$.

Proof. We first prove that

$$
(N(a \cup D) N(-a \cup D)) \cap \mathcal{S}_{\ell}(h) \subseteq G(h) .
$$

Let $\lambda \in(N(a \cup D) N(-a \cup D)) \cap \mathcal{S}_{\ell}(h)$. We show that $h \perp-\lambda h$ is hyperbolic. It is clear that $h \perp-\lambda h$ has even rank. Further $\operatorname{Nrd}(M(h \perp-\lambda h)) \equiv\left(a \lambda^{m}\right)^{4}$, and since $\left(a \lambda^{m}\right)^{2}$ is totally positive, by 1.8 it belongs to $\operatorname{Nrd}\left(D^{*}\right)$. Thus it follows that $h \perp-\lambda h$ has trivial Discriminant. Moreover since $\operatorname{disc}(h)$ is trivial, by 6.8 , it follows that the Clifford invariant of $h \perp-\lambda h$ is trivial. Since $\lambda \in \mathcal{S}_{\ell}(h)$, the form $h \perp-\lambda h$ has trivial signature as well.

By 6.9 we see that the Rost invariant $R(h \perp-\lambda h)=\left[(\lambda) \cup C_{+}(h)\right]$. We show that $\left[(\lambda) \cup C_{+}(h)\right]$ is trivial in $H^{3}(F, \mathbb{Z} / 4) / F^{*} \cup[D]$. Let $x \in F^{*}$ be such that $x \in$ $N(-a \cup D)$ and $\lambda x^{-1} \in N(a \cup D)$. We claim that $(\lambda) \cup\left[C_{+}(h)\right]=(x) \cup[D]$. In view of 6.2 , it suffices to check that at each $v \in \Omega$, we have $(\lambda) \cup\left[C_{+}(h)_{v}\right]=(x) \cup\left[D_{v}\right]$.

Suppose $v \in \Omega$ is such that $\lambda>_{v} 0$ and $x>_{v} 0$. In this case $(\lambda) \cup\left[C_{+}(h)_{v}\right]$ and $(x) \cup\left[D_{v}\right]$ are both trivial.

Suppose $\lambda>_{v} 0$ and $x<_{v} 0$. Then $\lambda x^{-1}<_{v} 0$. Since $\lambda x^{-1} \in N(a \cup D)$ and $x \in N(-a \cup D)$, in view of 6.6 both $(a) \cup[D]$ and $(-a) \cup[D]$ are split at $v$. Thus $-1 \in \operatorname{Nrd}\left(D_{v}\right)$ and hence $D$ is split at $v$. Thus both $(\lambda) \cup\left[C_{+}(h)_{v}\right]$ and $(x) \cup\left[D_{v}\right]$ are trivial in this case as well.

Now suppose that $\lambda<_{v} 0$ and $D_{v}$ is split. Since $\lambda \in \mathcal{S}_{l}(h)$, we conclude that $h_{v}$ is hyperbolic. Thus the Clifford invariant $\mathcal{C} \ell(h)_{v}=0$. Further, since $D_{v}$ is split and $h_{v}$ is hyperbolic, we have $C_{+}(h)_{v}=C_{-}(h)_{v}=0$. Thus we conclude that $C_{+}(h)_{v}$ is split and thus $(\lambda) \cup\left[C_{+}(h)_{v}\right]$ and $(x) \cup\left[D_{v}\right]$ are both zero.

Next, suppose that $\lambda<_{v} 0, D_{v}$ is not split and $x<_{v} 0$. Since $x \in N(-a \cup D)$, by $6.6(-a) \cup\left[D_{v}\right]=0$; i.e. $-a \in \operatorname{Nrd}\left(D_{v}\right)$. Hence $a<_{v} 0$. Since $D_{v}$ is not split and $a<_{v} 0$, by $6.1 C_{+}(h)_{v}$ is not split. Thus we conclude in this case that both $(\lambda) \cup\left[C_{+}(h)_{v}\right]$ and $(x) \cup\left[D_{v}\right]$ are non-zero and hence equal.

Now the only remaining case is when $\lambda<_{v} 0, D_{v}$ is not split and $x>_{v} 0$. In that case, $\lambda x^{-1}<_{v} 0$ and since $\lambda x^{-1} \in N(a \cup D)$, by 6.6 we have that $(a) \cup\left[D_{v}\right]=0$. Thus $a \in \operatorname{Nrd}\left(D_{v}\right)$ and hence $a>_{v} 0$. Since $D_{v}$ is non-split, by $6.1 C_{+}(h)_{v}$ is split. Thus both $(\lambda) \cup\left[C_{+}(h)_{v}\right]$ and $(x) \cup\left[D_{v}\right]$ are zero in this case.

We conclude therefore that $(\lambda) \cup\left[C_{+}(h)_{v}\right]=(x) \cup\left[D_{v}\right]$ for all $v \in \Omega$. Thus by 6.2 , we have $(\lambda) \cup\left[C_{+}(h)\right]=(x) \cup[D]$ and

$$
R(h \perp-\lambda h)=(\lambda) \cup\left[C_{+}(h)\right]=0 \in H^{3}(F, \mathbb{Z} / 4) / F^{*} \cup[D] .
$$

Since $\operatorname{vcd}(F) \leq 2$, by 6.3 we have that $h \perp-\lambda h$ is hyperbolic. Thus $\lambda \in G(h)$.

We now show the inclusion $G(h) \subseteq(N(a \cup D) N(-a \cup D)) \cap \mathcal{S}_{\ell}(h)$. It is clear that $G(h) \subseteq \mathcal{S}_{\ell}(h)$. We thus show that $G(h) \subseteq N(a \cup D) N(-a \cup D)$. Let $\lambda \in G(h)$. Then the the form $h \perp-\lambda h$ is hyperbolic and hence its Rost invariant $(\lambda) \cup\left[C_{+}(h)\right]$ is trivial. Thus there exists $x \in F^{*}$ such that $(\lambda) \cup\left[C_{+}(h)\right]=(x) \cup[D]$. By reading this equality locally at each $v \in \Omega$ and observing the sign pattern, we conclude that $x \in N(-a \cup D)$ and $\lambda x \in N(a \cup D)$. Therefore, $\lambda \in N(a \cup D) N(-a \cup D)$. This completes the proof. 
The following lemma will be used in the proof of 6.12 .

Lemma 6.11. Let $D$ be a central division algebra over $F$ and let $\tau$ be an orthogonal involution on $D$. Let $h$ be an even rank locally hyperbolic hermitian form over $(D, \tau)$ with $\operatorname{Disc}(h)=1$ and $\mathcal{C} \ell(h)=0$. Then $\operatorname{Hyp}(h)=F^{*}$.

Proof. Since the hermitian form $h$ has even rank, trivial discriminant and trivial Clifford invariant, there exists $\tilde{\xi} \in H^{1}\left(F, \operatorname{Spin}_{2 m}(D, \tau)\right)$ which maps to $[h] \in$ $H^{1}\left(F, \mathrm{U}_{2 m}(D, \tau)\right)$. Let $\mathcal{R}(\tilde{\xi}) \in H^{3}\left(F, \mu_{2}\right)$ be the Rost invariant of $\tilde{\xi}$. Let $L \in \mathcal{F}_{2}(F)$ be such that $\mathcal{R}\left(\tilde{\xi}_{L}\right)=(-1) \cup(-1) \cup(-x)$ for some $x \in L^{*}$. We claim that $\mathcal{R}\left(\tilde{\xi}_{L}\right)=(-x) \cup D_{L}$.

Let $\Omega_{L}$ be the set of orderings on $L$ and let $w \in \Omega_{L}$ be such that $D_{L w}$ is split. Then $\mathcal{R}\left(\tilde{\xi}_{L w}\right)=e_{3}\left(h_{L w}\right)$, where $e_{3}$ is the Arason invariant of quadratic forms. Since $h_{L w}$ is hyperbolic by hypothesis, we have $e_{3}\left(h_{L w}\right)=0$. Thus $\mathcal{R}\left(\tilde{\xi}_{L}\right)$ and $(-x) \cup D_{L}$ are both zero at $w$.

Now suppose $D_{L w}$ is not split. Then $D_{L w}=(-1) \cup(-1)$ and thus $\mathcal{R}\left(\tilde{\xi}_{L w}\right)=$ $(-1) \cup(-1) \cup(-x)=(-x) \cup D_{L w}$. Thus $\mathcal{R}\left(\tilde{\xi}_{L}\right)=(-x) \cup D_{L}$ at each $w \in \Omega_{L}$ and by $6.2, R\left(h_{L}\right)=0$. Therefore $h_{L}$ is a locally hyperbolic form with even rank, trivial Discriminant, trivial Clifford invariant and trivial Rost invariant. By 6.3 the form $h_{L}$ is hyperbolic. In view of this and 6.5 we conclude that $\operatorname{Hyp}(h)=F^{*}$.

The following proposition gives an explicit description of the group $\operatorname{Hyp}(h)$.

Proposition 6.12. We have $\operatorname{Hyp}(h)=\left(N(a \cup D) \cap \mathcal{S}_{\ell}(h)\right) .\left(N(-a \cup D) \cap \mathcal{S}_{\ell}(h)\right)$.

Proof. We first prove that $\operatorname{Hyp}(h) \subseteq\left(N(a \cup D) \cap \mathcal{S}_{\ell}(h)\right) .\left(N(-a \cup D) \cap \mathcal{S}_{\ell}(h)\right)$. Let $L / F$ be a finite extension such that $h_{L}$ is hyperbolic. Then $\operatorname{Disc}\left(h_{L}\right)$ is trivial in $L^{*} / \operatorname{Nrd}\left(D_{L}^{*}\right)^{2}$ and hence either $a \in \operatorname{Nrd}\left(D_{L}^{*}\right)$ or $-a \in \operatorname{Nrd}\left(D_{L}^{*}\right)$; i.e. either $N\left(a \cup D_{L}\right)=L^{*}$ or $N\left(-a \cup D_{L}\right)=L^{*}$. We clearly have $\mathcal{S}_{\ell, L}(h)=L^{*}$. Thus

$$
\left(N\left(a \cup D_{L}\right) \cap \mathcal{S}_{\ell, L}(h)\right) .\left(N\left(-a \cup D_{L}\right) \cap \mathcal{S}_{\ell, L}(h)\right)=L^{*} .
$$

Clearly $N_{L / F}\left(N\left(a \cup D_{L}\right)\right) \subseteq N(a \cup D)$ and $N_{L / F}\left(N\left(-a \cup D_{L}\right)\right) \subseteq N(-a \cup D)$. Further as in [KMRT, Prop. 12.21], $N_{L / F}\left(\mathcal{S}_{\ell, L}(h)\right) \subseteq \mathcal{S}_{\ell}(h)$. Thus

$$
\begin{aligned}
N_{L / F}\left(L^{*}\right) & \subseteq N_{L / F}\left(\left(N\left(a \cup D_{L}\right) \cap \mathcal{S}_{\ell, L}(h)\right) .\left(N\left(-a \cup D_{L}\right) \cap \mathcal{S}_{\ell, L}(h)\right)\right) \\
& \subseteq\left(N(a \cup D) \cap \mathcal{S}_{\ell}(h)\right) .\left(N(-a \cup D) \cap \mathcal{S}_{\ell}(h)\right) .
\end{aligned}
$$

Since $N_{L / F}\left(L^{*}\right)$ generate $\operatorname{Hyp}(h)$ as $L$ runs over extensions where $h$ is hyperbolic, it follows that $\operatorname{Hyp}(h) \subseteq\left(N(a \cup D) \cap \mathcal{S}_{\ell}(h)\right) .\left(N(-a \cup D) \cap \mathcal{S}_{\ell}(h)\right)$.

To complete the proof, we show that $N(a \cup D) \cap \mathcal{S}_{\ell}(h) \subseteq \operatorname{Hyp}(h)$. The inclusion $N(-a \cup D) \cap \mathcal{S}_{\ell}(h) \subseteq \operatorname{Hyp}(h)$ follows in a similar manner. Let $\lambda \in N(a \cup D) \cap$ $\mathcal{S}_{\ell}(h)$. By $6.10, \lambda \in G(h)$. Let $K=F(\sqrt{-\lambda})$. Since $\lambda \in N(a \cup D)$, by 6.6 $(\lambda) \cup(a) \cup[D]=0 \in H^{4}\left(F, \mu_{2}\right)$. Thus $(-1) \cup(a) \cup\left[D_{K}\right]=0 \in H^{4}\left(K, \mu_{2}\right)$. By 1.15, $(a) \cup\left[D_{K}\right]=0 \in H^{3}\left(K, \mu_{2}\right)$. Hence $a \in \operatorname{Nrd}\left(D_{K}\right)$ and $\operatorname{Disc}(h)_{K}=1$.

Let $w$ be an ordering on $K$. Since $\lambda<_{w} 0$ and $\lambda \in G\left(h_{K}\right)$, the form $h_{K}$ is locally hyperbolic. Thus the Clifford invariant $\mathcal{C} \ell(h)_{K}$ is trivial at $w$. Therefore, if $D_{K w}$ is split, then $C_{+}(h)_{K w}=C_{-}(h)_{K w}=0$. If $D_{K w}$ is not split, then in view of $1.8, a>_{w} 0$ as $a \in \operatorname{Nrd}\left(D_{K}\right)$. By $6.1, C_{+}(h)_{K w}$ is split. We have thus shown that $C_{+}\left(h_{K}\right)$ is locally split. By 1.8, it follows that $\operatorname{Nrd}\left(C_{+}\left(h_{K}\right)\right)=K^{*}$. Let $L / K$ be a finite extension and let $\alpha \in L^{*}$ be such that $\sqrt{-\lambda}=N_{L / K}(\alpha)$ and 
$C_{+}\left(h_{L}\right)=0$. Then $h_{L}$ is an even rank locally hyperbolic form with $\operatorname{Disc}\left(h_{L}\right)=1$ and $C_{+}\left(h_{L}\right)=0$. By $6.11, \operatorname{Hyp}\left(h_{L}\right)=L^{*}$. Thus

$$
\sqrt{-\lambda}=N_{L / K}(\alpha) \in N_{L / K}\left(\operatorname{Hyp}\left(h_{L}\right)\right) \subseteq \operatorname{Hyp}\left(h_{K}\right) .
$$

Taking norm from $K / F$ we have $\lambda \in \operatorname{Hyp}(h)$. Thus $N(a \cup D) \cap \mathcal{S}_{\ell}(h) \subseteq \operatorname{Hyp}(h)$.

With the notation as above, we have following corollaries.

Corollary 6.13. If $h$ is locally hyperbolic, then

$$
\operatorname{Hyp}(h)=N(a \cup D) \cdot N(-a \cup D)=G(h) .
$$

Proof. Since $h$ is locally hyperbolic, $\mathcal{S}_{\ell}(h)=F^{*}$. From 6.10 and 6.12 , it is clear that $\operatorname{Hyp}(h)=N(a \cup D) \cdot N(-a \cup D)=G(h)$.

Corollary 6.14. If $h$ has trivial Discriminant, then $\operatorname{Hyp}(h)=\mathcal{S}_{\ell}(h)=G(h)$.

Proof. Since $\operatorname{Disc}(h)=1$, it follows that either $N(a \cup D)=F^{*}$ or $N(-a \cup D)=F^{*}$. In either case, it is immediate from 6.10 and 6.12, that $\operatorname{Hyp}(h)=\mathcal{S}_{\ell}(h)=G(h)$.

Let $A$ be a central simple algebra over $F$ with an orthogonal involution $\sigma$. Suppose $\operatorname{disc}(\sigma)=1$ and $\mathrm{C}(A, \sigma)=\mathrm{C}_{+}(A, \sigma) \times \mathrm{C}_{-}(A, \sigma)$. We have the following extension of 6.13

Proposition 6.15. Let $F$ be a field with $\operatorname{char}(F) \neq 2$. Let $(A, \sigma)$ be a central simple algebra of even degree over $F$ with an orthogonal involution. Let $\operatorname{deg}(A) \equiv 0(4)$ and $\operatorname{disc}(\sigma)=1$. Then $\operatorname{Hyp}(A, \sigma) \subseteq \operatorname{Nrd}\left(\mathrm{C}_{+}(A, \sigma)\right) \operatorname{Nrd}\left(\mathrm{C}_{-}(A, \sigma)\right)$. Further if $\operatorname{vcd}(F) \leq 2$ and $\sigma$ is locally hyperbolic, then

$$
\operatorname{Hyp}(A, \sigma)=\operatorname{Nrd}\left(\mathrm{C}_{+}(A, \sigma)\right) \operatorname{Nrd}\left(\mathrm{C}_{-}(A, \sigma)\right)=G(A, \sigma) .
$$

Proof. The first assertion follows from the fact that over any extension $L / F$ where $\sigma$ is hyperbolic, either $\mathrm{C}_{+}\left(A_{L}, \sigma_{L}\right)$ or $\mathrm{C}_{-}\left(A_{L}, \sigma_{L}\right)$ is split [KMRT, Prop. 12.21].

Suppose $\operatorname{vcd}(F) \leq 2$ and $\sigma$ is locally hyperbolic. Let $\lambda \in \operatorname{Nrd}\left(\mathrm{C}_{+}(A, \sigma)\right)$. Let $L / F$ be a finite extension such that $\lambda \in N_{L / F}\left(L^{*}\right)$ and $\mathrm{C}_{+}\left(A_{L}, \sigma_{L}\right)$ is split. We show that $\operatorname{Hyp}\left(A_{L}, \sigma_{L}\right)=L^{*}$. In view of 2.3 , replacing $L$ by a quadratic tower, we may assume that $A_{L} \simeq M_{2 r}(H)$ for some quaternion algebra $H$ over $L$. Let $\tau$ be an orthogonal involution on $H$ and let $h$ be a hermitian form over $(H, \tau)$ such that $\sigma_{L}$ is adjoint to $h$. Let $M_{h} \in M_{2 r}(H)$ represent $h$ and $\operatorname{Nrd}\left(M_{h}\right)=a^{2}$ for some $a \in L^{*}$. Let $w$ be an ordering on $L$ such that $H_{w}$ is not split. Since $C_{+}(h)=C_{+}\left(A_{L}, \sigma_{L}\right)$ is split, by $6.1 a>_{w} 0$. Thus $(a) \cup[H]=0 \in H^{3}\left(L, \mu_{2}\right)$ and $N(a \cup H)=L^{*}$. In view of 6.13, $\operatorname{Hyp}\left(A_{L}, \sigma_{L}\right)=\operatorname{Hyp}(h)=N(a \cup H) \cdot N(-a \cup H)=L^{*}$. Thus $\operatorname{Hyp}\left(A_{L}, \sigma_{L}\right)=$ $L^{*}$. Taking norms from $L / F$ we have $\lambda \in N_{L / F}\left(L^{*}\right)=N_{L / F}\left(\operatorname{Hyp}\left(A_{L}, \sigma_{L}\right)\right) \subseteq$ $\operatorname{Hyp}(A, \sigma)$. Thus $\operatorname{Nrd}\left(\mathrm{C}_{+}(A, \sigma)\right) \subseteq \operatorname{Hyp}(A, \sigma)$.

The inclusion $\operatorname{Nrd}\left(\mathrm{C}_{-}(A, \sigma)\right) \subseteq \operatorname{Hyp}(A, \sigma)$ follows from a similar argument. We therefore conclude that $\operatorname{Hyp}(A, \sigma)=\operatorname{Nrd}\left(\mathrm{C}_{+}(A, \sigma)\right) \operatorname{Nrd}\left(\mathrm{C}_{-}(A, \sigma)\right)$.

To complete the proof we show that $G(A, \sigma) \subseteq \operatorname{Nrd}\left(\mathrm{C}_{+}(A, \sigma)\right) \operatorname{Nrd}\left(\mathrm{C}_{-}(A, \sigma)\right)$. Let $\lambda \in G(A, \sigma)$. Then the hermitian form $\langle 1,-\lambda\rangle$ is hyperbolic. Hence the Rost invariant $R(\langle 1,-\lambda\rangle)$ is trivial. As in the proof of $6.9, R(\langle 1,-\lambda\rangle)=(\lambda) \cup$ $\left[\mathrm{C}_{+}(A, \sigma)\right]$. Since the Rost invariant is trivial, there exists $x \in F^{*}$ such that $(\lambda) \cup$ $\left[\mathrm{C}_{+}(A, \sigma)\right]=(x) \cup[A]$. If for an ordering $v$ on $F$, the algebra $A_{v}$ is split, then $h_{v}$ being hyperbolic, $\mathrm{C}_{+}(A, \sigma)_{v}$ and $\mathrm{C}_{+}(A, \sigma)_{v}$ are both split. If $A_{v}$ is not split and $x<_{v} 0$, then $\mathrm{C}_{+}(A, \sigma)_{v}$ is not split. Hence $\mathrm{C}_{-}(A, \sigma)_{v}$ is split. Thus $x \in$ 
$\operatorname{Nrd}\left(\mathrm{C}_{-}(A, \sigma)\right)$ and a similar argument gives $\lambda x \in \operatorname{Nrd}\left(\mathrm{C}_{+}(A, \sigma)\right)$. Hence $\lambda=$ $\lambda x . x^{-1} \in \operatorname{Nrd}\left(\mathrm{C}_{+}(A, \sigma)\right) \operatorname{Nrd}\left(\mathrm{C}_{+}(A, \sigma)\right)$. We have thus shown that

$$
G(A, \sigma) \subseteq \operatorname{Hyp}(A, \sigma)=\operatorname{Nrd}\left(\mathrm{C}_{+}(A, \sigma)\right) \operatorname{Nrd}\left(\mathrm{C}_{-}(A, \sigma)\right) .
$$

The inclusion $\operatorname{Hyp}(A, \sigma) \subseteq G(A, \sigma)$ follows from 1.1 and this completes the proof.

Theorem 6.16. Let $\operatorname{vcd}(F) \leq 2$ and let $A$ be a central simple algebra over $F$ with $\operatorname{deg}(A)$ even and an involution $\sigma$ of orthogonal type. If $\operatorname{disc}(\sigma)=1$ and $\sigma$ is locally hyperbolic, then $G(A, \sigma)=\operatorname{Hyp}(A, \sigma) \cdot F^{* 2}$.

Proof. Let $\operatorname{deg}(A)=2 n$. Suppose $n$ is odd. Since $\sigma$ is locally hyperbolic, the algebra $A$ is locally split and by 6.4 the results holds. We can thus assume that $n$ is even. In this case we are through by 6.15 .

\section{Fields With $\operatorname{vcd}(F) \leq 2$ SATisfying SAP}

Let $F$ be a field with orderings and let $\Omega$ denote the set of orderings on $F$. Given $a \in F^{*}$, we define the corresponding Harrison set $\Omega_{a}$ as follows:

$$
\Omega_{a}:=\left\{v \in \Omega: a>_{v} 0\right\} .
$$

The set $\Omega$ has Harrison topology for which $\left\{\Omega_{a}: a \in F^{*}\right\}$ is a sub-basis. With this topology, $\Omega$ is a Hausdorff, compact and totally disconnected space. We say that $F$ has strong approximation property (SAP), if every closed and open set of $\Omega$ is of the form $\Omega_{a}$ for some $a \in F^{*}$. A quadratic form $q$ is said to be weakly isotropic, if for some positive integer $s$, the $s$-fold orthogonal sum $s . q=\perp_{s} q$ is isotropic. Combining [ELP, Th. C] and [P, Satz. 3.1] we have the following

Theorem 7.1. A field $F$ with orderings has SAP if and only if for every $a, b \in F^{*}$, the quadratic form $\langle 1, a, b,-a b\rangle$ is weakly isotropic.

In what follows, for $a_{1}, a_{2}, \cdots, a_{r} \in F^{*}$ the notation $\left\langle\left\langle a_{1}, a_{2}, \cdots, a_{r}\right\rangle\right\rangle$ will denote the $r$-fold Pfister form $\left\langle 1,-a_{1}\right\rangle \otimes\left\langle 1,-a_{2}\right\rangle \otimes \cdots \otimes\left\langle 1,-a_{r}\right\rangle$. For a quadratic form $q$, we denote by $D(q)$ the set of elements of $F^{*}$ represented by $q$. We remark that if $q=\langle 1,-a,-b, a b\rangle$, then $D(q)=\operatorname{Nrd}\left(H^{*}\right)$, where $H$ is the quaternion algebra $(a, b)$ over $F$. Set $\Omega(H)=\left\{v \in \Omega: H \otimes_{F} F_{v}\right.$ is split $\}$. The following lemma is recorded in $[\mathrm{Ga}]$.

Lemma 7.2. Let $F$ be a field with orderings. Let $a, b \in F^{*}$. Let $q_{1}=\langle\langle-1,-a\rangle\rangle$, $q_{2}=\langle\langle-1, a\rangle\rangle$ and $H=(a, b)$. Suppose there does not exist $c \in F^{*}$ such that $\Omega_{c}=\Omega(H)$. Then $-b \notin D\left(q_{1}\right) D\left(q_{2}\right)$.

Proof. Suppose $-b \in D\left(q_{1}\right) D\left(q_{2}\right)$ and let $x_{1} \in D\left(q_{1}\right)$ and $x_{2} \in D\left(q_{2}\right)$ be such that $-b=x_{1} x_{2}$. Then $q_{1} \perp b q_{2}$ is isotropic and hence $2\langle 1, a, b,-a b\rangle \simeq q_{0} \perp \mathbb{H}$ for some Albert form $q_{0}$ and $\mathbb{H} \simeq\langle 1,-1\rangle$. We have

$$
C\left(q_{0}\right)=C\left(q_{1} \perp b q_{2}\right)=(-1,-1)=C(4\langle 1\rangle \perp \mathbb{H}) \in \operatorname{Br}(F) .
$$

Therefore by [KMRT, Prop. 16.3], $q_{0} \simeq 4\langle c\rangle \perp \mathbb{H}$ for some $c \in F^{*}$. It is easy to see that $\Omega_{c}=\Omega(H)$, which contradicts the hypothesis. Thus $-b \notin D\left(q_{1}\right) D\left(q_{2}\right)$.

Lemma 7.3. Let $F$ be a field for which $I^{3}(F)$ is torsion-free. Let $a, b \in F^{*}$. Let $q_{1}=\langle\langle-1,-a\rangle\rangle, q_{2}=\langle\langle-1, a\rangle\rangle$ and $H=(a, b)$. If $-b \notin D\left(q_{1}\right) D\left(q_{2}\right)$, then there is no element $c \in F^{*}$ with $\Omega_{c}=\Omega(H)$. 
Proof. Suppose there is an element $c \in F^{*}$ such that $\Omega_{c}=\Omega(H)$. Let $q^{\prime}=$ $\langle 1, a, b,-a b,-c,-c\rangle$. For $v \in \Omega$ if $c<_{v} 0$, then by the choice of $c$ we have $a<_{v} 0$ and $b<_{v} 0$. This implies that the form $q^{\prime}$ is hyperbolic at $v$. If $c>_{v} 0$, then again by the choice of $c$, either $a>_{v} 0$ or $b>_{v} 0$ and in either case $q^{\prime}$ is hyperbolic at $v$. We thus conclude that $q^{\prime}$ is locally hyperbolic. Clearly $q^{\prime} \in I^{2}(F)$, therefore $2 q^{\prime} \in I^{3}(F)$. Since $2 q^{\prime}$ is an even rank quadratic form with trivial signature, it is hyperbolic at each $F_{v}, v \in \Omega$. Thus by Pfister's local-global principle [L, Th. VIII.4.1], $2 q^{\prime}$ is a torsion element in the Witt group $W(F)$. By the hypothesis, $I^{3}(F)$ is torsion-free. Thus $2 q^{\prime}=2\langle 1, a, b,-a b,-c,-c\rangle$ is hyperbolic. Therefore the form $q_{1} \perp b q_{2}$ is isotropic, which implies that $-b \in D\left(q_{1}\right) D\left(q_{2}\right)$. This is a contradiction to the hypothesis.

Combining 7.2 and 7.3 above, we get the following

Corollary 7.4. Let $I^{3}(F)$ be torsion-free. Let $a, b \in F^{*}$ and $q_{1}=\langle\langle-1,-a\rangle\rangle$, $q_{2}=\langle\langle-1, a\rangle\rangle$ and $H=(a, b)$. Then $-b \in D\left(q_{1}\right) D\left(q_{2}\right)$ if and only if there exists $c \in F^{*}$ such that $\Omega_{c}=\Omega(H)$.

Using the results above, we have thus derived

Corollary 7.5. Let $F$ be a field with $I^{3}(F)$ torsion-free. Then the following statements are equivalent:

(i) For all $a \in F^{*}$ we have $D(\langle\langle-1,-a\rangle\rangle) D(\langle\langle-1, a\rangle\rangle)=F^{*}$.

(ii) The field $F$ has SAP.

(iii) Given a quaternion algebra $H=(a, b)$ over $F$, there exists an element $c \in F^{*}$ such that $\Omega_{c}=\Omega(H)$.

Lemma 7.6. Let $I^{3}(F)$ be torsion-free, and $H$ be a quaternion algebra over $F$. Then $\operatorname{Nrd}\left(H^{*}\right)=\left\{\lambda \in F^{*}: \lambda>_{v} 0\right.$ at each $\left.v \in \Omega \backslash \Omega(H)\right\}$.

Proof. Let $n_{H}$ denote the norm form of the quaternion algebra $H$. Since $I^{3}(F)$ is torsion-free, for $\lambda \in F^{*},\langle 1,-\lambda\rangle \otimes n_{H}=0 \in I^{3}(F)$ if and only if for all $v \in$ $\Omega,\langle 1,-\lambda\rangle \otimes n_{H}=0 \in I^{3}\left(F_{v}\right)$. In other words, $\lambda \in \operatorname{Nrd}\left(H^{*}\right)$ if and only if $\lambda \in \operatorname{Nrd}\left(H \otimes F_{v}\right)^{*}$ at each $v \in \Omega$. This is equivalent to saying that $\lambda>_{v} 0$ if $v \in \Omega \backslash \Omega(H)$, and the lemma follows.

Lemma 7.7. Suppose $I^{3}(F)$ is torsion-free, $F$ has orderings and satisfies SAP. Then for every $a, b \in F^{*}$ we have $\operatorname{Nrd}(a, b)^{*} . \operatorname{Nrd}(-a, b)^{*}=F^{*}$.

Proof. Let $H_{1}=(a, b)$ and $H_{2}=(-a, b)$. Since $F$ has SAP, the closed and open set $\Omega \backslash \Omega\left(H_{1}\right)=\Omega_{x}$ for some $x \in F^{*}$. Similarly $\Omega \backslash \Omega\left(H_{1}\right)=\Omega_{y}$ for some $y \in F^{*}$. By 7.6, $\operatorname{Nrd}\left(H_{1}^{*}\right)=\operatorname{Nrd}(-1,-x)$ and $\operatorname{Nrd}\left(H_{2}^{*}\right)=\operatorname{Nrd}(-1,-y)^{*}$. Since at a given ordering $v \in \Omega$, at least one of $H_{1}$ and $H_{2}$ is split, $\Omega_{x} \cap \Omega_{y}=\phi$. Thus $\Omega_{x} \subseteq \Omega_{-y}$. Now using 7.6, we conclude that $\operatorname{Nrd}(-1, y)^{*} \subseteq \operatorname{Nrd}(-1,-x)^{*}$. Thus in view of $7.5, F^{*}=\operatorname{Nrd}(-1, y)^{*} \operatorname{Nrd}(-1,-y)^{*} \subseteq \operatorname{Nrd}(-1,-x)^{*} \operatorname{Nrd}(-1,-y)^{*}$. This proves $\operatorname{Nrd}\left(H_{1}\right)^{*} \cdot \operatorname{Nrd}\left(H_{2}\right)^{*}=F^{*}$.

Detlev Hoffmann has suggested the following more direct proof of 7.7.

Lemma 7.8. Let $I^{3}(F)$ be torsion-free, where $F$ has orderings and satisfies SAP. Then for every $a, b \in F^{*}$ we have $\operatorname{Nrd}(a, b)^{*} \cdot \operatorname{Nrd}(-a, b)^{*}=F^{*}$. 
Proof. Let $H_{1}=(a, b)$ and $H_{2}=(-a, b)$. Let $X_{i}=\Omega \backslash \Omega\left(H_{i}\right), i=1,2$, and let $\lambda \in F^{*}$ be arbitrary. Since $F$ has SAP, there exists $x \in F^{*}$ such that $X_{1} \cup\left(X_{2} \cap\right.$ $\left.\Omega_{\lambda}\right)=\Omega_{x}$. Using the hypothesis that $I^{3}(F)$ is torsion-free and the observation that $X_{1} \cap X_{2}=\phi$, one can conclude that $x \in \operatorname{Nrd}\left(H_{1}\right)^{*}$ and $\lambda x^{-1} \in \operatorname{Nrd}\left(H_{2}\right)^{*}$. Thus $\lambda=x \lambda x^{-1} \in \operatorname{Nrd}\left(H_{1}\right)^{*} . \operatorname{Nrd}\left(H_{2}\right)^{*}$.

From now on, in this section the field $F$ satisfies $\operatorname{vcd}(F) \leq 2$. We say that a field $F$ satisfies SAP for quadratic towers if each quadratic tower $L \in \mathcal{F}_{2}(F)$ has $\operatorname{SAP}$.

Proposition 7.9. Let $\operatorname{vcd}(F) \leq 2$ and $F$ has SAP for quadratic towers. Let $h$ be a locally hyperbolic hermitian form of even rank over a central-division algebra $D$ with an orthogonal involution $\tau$. Let $\operatorname{disc}(h)=1$. Then we have $\operatorname{Hyp}(h)=F^{*}$.

Proof. Let $L \in \mathcal{F}_{2}(F)$ be such that $D_{L} \sim(-1,-x)$ for some $x \in L^{*}$. Since $h_{L}$ is locally hyperbolic, by 6.13 we have $\operatorname{Hyp}\left(h_{L}\right)=N\left(a \cup D_{L}\right) \cdot N\left(-a \cup D_{L}\right)$. Clearly $N\left(a \cup D_{L}\right)=\operatorname{Nrd}(a,-x)^{*}$ and $N\left(-a \cup D_{L}\right)=\operatorname{Nrd}(-a,-x)^{*}$. Since $F$ has SAP for quadratic towers, so does $L$. Thus by 7.7 , we have

$$
\operatorname{Nrd}(a,-x)^{*} \cdot \operatorname{Nrd}(-a,-x)^{*}=L^{*}
$$

and we conclude that $\operatorname{Hyp}\left(h_{L}\right)=L^{*}$. In view of this and 2.4 we have $\operatorname{Hyp}(h)=$ $F^{*}$.

Proposition 7.10. Let $\operatorname{vcd}(F) \leq 2$ and $F$ has SAP with respect to quadratic towers. Let $A$ be a central simple algebra of even degree over $F$ and let $\sigma$ be a locally hyperbolic involution on $A$ with $\operatorname{disc}(\sigma)=1$. Then $\operatorname{Hyp}(A, \sigma)=F^{*}$.

Proof. Let $L \in \mathcal{F}_{2}(F)$ be such that $A_{L} \sim(-1,-x)$ for some $x \in L^{*}$. Let $H=$ $(-1,-x)$. Then $A_{L}=M_{r}(H)$ for some positive integer $r$. Let $\tau$ be an orthogonal involution on $H$ and let $h$ be a hermitian form over $(H, \tau)$ such that $\sigma_{L}$ is adjoint to $h$. Then $\operatorname{Hyp}\left(A_{L}, \sigma_{L}\right)=\operatorname{Hyp}(h)$.

First assume that $r$ is even. Then it follows from [KMRT, Prop. 7.3(1)] that $\operatorname{disc}\left(\sigma_{L}\right)=\operatorname{disc}(h)=1$. Since $\sigma_{L}$ is locally hyperbolic, the hermitian form $h$ is locally hyperbolic. Thus $h$ is a locally hyperbolic form of even rank and trivial discriminant over $(H, \tau)$. Therefore in view of 7.9 we have $\operatorname{Hyp}\left(A_{L}, \sigma_{L}\right)=\operatorname{Hyp}(h)=$ $L^{*}$.

Now suppose $r$ is odd. Since $\sigma_{L}$ is locally hyperbolic, the hermitian form $h$ is locally hyperbolic. Thus the quaternion algebra $H$ is locally split and by 1.8 we have $\operatorname{Nrd}(H)^{*}=L^{*}$. Let $\lambda \in L^{*}$. Let $L_{i} \in \mathcal{F}_{2}(L)$ be such that each $H_{L_{i}}$ is split and $\lambda=\prod_{i} N_{L_{i} / L}\left(\lambda_{i}\right)$, where $\lambda_{i} \in L_{i}^{*}$. Then $h_{L_{i}}$ is a locally hyperbolic quadratic form over $L_{i}$ with even rank and trivial discriminant. Thus by 5.2, we have $\operatorname{Hyp}\left(h_{L_{i}}\right)=L_{i}^{*}$. Therefore

$$
\lambda \in \prod_{i} N_{L_{i} / L}\left(\operatorname{Hyp}\left(H_{L_{i}}\right)\right) \subseteq \operatorname{Hyp}(h)
$$

and hence $\operatorname{Hyp}(h)=L^{*}$.

Thus it follows that if $L \in \mathcal{F}_{2}(F)$ is such that $A_{L} \sim(-1,-x)$ for some $x \in L^{*}$, then $\operatorname{Hyp}\left(A_{L}, \sigma_{L}\right)=\operatorname{Hyp}(h)=L^{*}$. Therefore in view of 2.4, we have $\operatorname{Hyp}(A, \sigma)=$ $F^{*}$. This completes the proof.

Theorem 7.11. Let $\operatorname{vcd}(F) \leq 2$ and $F$ has SAP for quadratic towers. Let $A$ be a central simple algebra of degree $2 n$ over $F$ and let $\sigma$ be an orthogonal involution on $A$ with $\operatorname{disc}(\sigma)=1$. Then $\operatorname{Hyp}(A, \sigma) \cdot F^{* 2}=G_{+}(A, \sigma)$. 
Proof. Let $\lambda \in G_{+}(A, \sigma)$. Then $\lambda=\sigma(a) a$ for some $a \in A^{*}$ with $\operatorname{Nrd}(a)=\lambda^{n}$. Let $K=F(\sqrt{-\lambda})$. First suppose that $n$ is odd. Then $\lambda \in \operatorname{Nrd}\left(A^{*}\right)$ and thus $\lambda>_{v} 0$ at those $v \in \Omega$ where $A_{v}$ is not split. If $A_{v}$ is split, then $\lambda>{ }_{v} 0$ at those $v \in \Omega$ where $\operatorname{sgn}\left(\sigma_{v}\right) \neq 0$. Then $A_{K}$ is locally split and $\sigma_{K}$ is locally hyperbolic.

Now suppose that $n$ is even and let $v \in \Omega$ be such that $\sigma_{v}$ is not hyperbolic. Then by (Theorem 3.7, Chapter 10, [Sc]), $A_{v}$ is split and $\sigma_{v}$ is adjoint to a non-hyperbolic quadratic form $q$ over $F_{v}$ such that $q \simeq \lambda q$. Thus we conclude that $\lambda>_{v} 0$ at those orderings $v \in \Omega$ where $\sigma_{v}$ is not hyperbolic. Then $\sigma_{K}$ is locally hyperbolic.

Thus in either case, by 7.10 we have that $\operatorname{Hyp}\left(A_{K}, \sigma_{K}\right)=K^{*}$. Taking norm of $\sqrt{-\lambda}$ from $K / F$, we conclude that $\lambda \in \operatorname{Hyp}(A, \sigma)$ and therefore $G_{+}(A, \sigma) \subseteq$ $\operatorname{Hyp}(A, \sigma)$. By 1.1 we have $\operatorname{Hyp}(A, \sigma) \cdot F^{* 2} \subseteq G_{+}(A, \sigma)$, and hence we conclude that $G_{+}(A, \sigma)=\operatorname{Hyp}(A, \sigma) \cdot F^{* 2}$.

Theorem 7.12. Let $\operatorname{vcd}(F) \leq 2$ and $(A, \sigma)$ be an algebra of type ${ }^{2} D_{n}$ over $F$. Let $F$ have SAP for quadratic towers. Then we have $G_{+}(A, \sigma)=\operatorname{Hyp}(A, \sigma) . F^{* 2}$.

Proof. Let $\operatorname{disc}(\sigma)=d$ and let $\lambda \in G_{+}(A, \sigma)$. By 1.3, we have $G_{+}(A, \sigma) \subseteq$ $N_{L / K}\left(L^{*}\right)$, where $L=F(\sqrt{d})$. As in the proof of $7.11, \sigma_{F(\sqrt{-\lambda})}$ is locally hyperbolic. Let $M=L(\sqrt{-\lambda})$. By the biquadratic lemma [W, Lemma 2.14], it follows that there exist $x \in M^{*}$ and $y \in F^{*}$ such that $\lambda=N_{M / F}(x) y^{2}$. It is clear that $\operatorname{disc}\left(\sigma_{M}\right)=1$ and $\sigma_{M}$ is locally hyperbolic. Thus by 7.10 we have $\operatorname{Hyp}\left(A_{M}, \sigma_{M}\right)=$ $M^{*}$ and we easily see that $\lambda y^{-2} \in \operatorname{Hyp}(A, \sigma)$. Thus $G_{+}(A, \sigma) \subseteq \operatorname{Hyp}(A, \sigma) . F^{* 2}$. Since by $1.1 \operatorname{Hyp}(A, \sigma) \cdot F^{* 2} \subseteq G_{+}(A, \sigma)$, we conclude that $G_{+}(A, \sigma)=\operatorname{Hyp}(A, \sigma) \cdot F^{* 2}$.

Corollary 7.13. Suppose $\operatorname{vcd}(F) \leq 2$, and $F$ has SAP for quadratic towers. Let the group $\mathbf{P S i m}_{+}(A, \sigma)$ be of type ${ }^{1} D_{n}$ or ${ }^{2} D_{n}$. Then $\mathbf{P S i m}_{+}(A, \sigma)(F) / R=0$.

Since number fields satisfy the conditions of 7.13 , we have

Corollary 7.14. Let $F$ be a number field and let $\mathbf{P S i m}_{+}(A, \sigma)$ be of type ${ }^{1} D_{n}$ or ${ }^{2} D_{n}$. Then $\mathbf{P S i m}_{+}(A, \sigma)(F) / R=0$.

Remark. It is a well known fact that SAP is not preserved under field extensions. As Detlev Hoffmann has pointed out to us, there are examples of fields $F$ with $\operatorname{vcd}(F)=2$ and quadratic extensions $E / F$ such that $F$ satisfies SAP but not $E$. Thus the condition 'SAP with respect to quadratic towers' is not redundant in 7.12.

Hoffmann's example is the following: One can construct a formally real field $k$ with the following properties: (i) $k$ has no extension of odd degree. (ii) There is only one ordering on $k$. (iii) The $u$-invariant of $k$ is 2 . Let $\alpha \in k^{*} \backslash k^{* 2}$ be a sum of two squares. Let $F=k((X))$ and $E=F(\sqrt{\alpha})$. Then $\operatorname{vcd}(F)=2$ and $F$ has SAP but not $E$.

Combining together the results of $\S 4, \S 5$ and $\S 7$, we have

Theorem 7.15. Let $F$ be a field with $\operatorname{vcd}(F) \leq 2$. Let $G$ be a classical group of adjoint type defined over $F$. Then,

(i) If $G$ does not contain a factor of type $D_{n}$, then $G(F) / R=0$.

(ii) If $F$ satisfies SAP for quadratic towers, then $G(F) / R=0$.

Proof. If $F$ does not have orderings, by 1.17 we have $\operatorname{cd}(F) \leq 2$ and we are through by 3.7. Thus we assume that $F$ has orderings. As in the proof of 3.7 , it suffices to prove the theorem for absolutely simple adjoint groups defined over $F$. In view 
of 1.1, assertion (i) follows from 1.4, 4.2 and 5.13. Assertion (ii) of the theorem follows immediately from assertion (i) and 7.13.

\section{REFERENCES}

[A1] Arason J., Cohomologische Invarianten quadratischer Formen, J. Algebra 36(1975), pp 448 - 491. MR0389761 (52:10592)

[A2] Arason J., A proof of Merkurjev's theorem, Quadratic and hermitian forms, CMS Conference Proceedings 4 (1984), pp 121 - 130. MR0776449 (86f:11029)

[AEJ] Arason J., Elman R. and Jacob B., Fields of cohomological 2-dimension three, Math. Ann. 274 (1986), pp 649 - 657. MR0848510 (87m:12006)

[Ar] Artin M., Dimension cohomologique: premiers résultats, Théorie des Topos et Cohomologie Etale des Schémes, Lecture Notes in Mathematics 305(1963-64), pp 43 - 63.

[B] Bartels H.-J., Invarianten hermitescher Formen über Schiefkörpern, Math. Ann. 215(1975), pp 269 - 288. MR0419353 (54:7374)

[BP1] Bayer-Fluckiger E. and Parimala R., Galois cohomology of classical groups over fields of cohomological dimension $\leq 2$, Inventiones mathematicae 122(1995), pp 195-229. MR1358975 (96i:11042)

[BP2] Bayer-Fluckiger E. and Parimala R., Classical groups and the Hasse principle, Ann. of Math. 147(1998), pp 651-693. MR1637659 (99g:11055)

[BMPS] Bayer-Fluckiger E., Monsurrò M., Parimala R. and Schoof R., Trace forms of $G$-Galois algebras in virtual cohomological dimension 1 and 2, Pacific J. Math 217(2004), pp 29 - 43. MR2105764 (2005i:12005)

[CM] Chernousov V. and Merkurjev A., $R$-equivalence and Special Unitary Groups, J. Algebra 209(1998), pp 175 - 198. MR1652122 (99m:20101)

[CTS] Colliot-Thélène J.-L. and Sansuc J.-J., La R-équivalence sur les tores, Ann. scient. Éc. Norm. Sup., $4^{e}$ série 10(1977), pp 175-230. MR0450280 (56:8576)

[CTGP] Colliot-Thélène J.-L., Gille P. and Parimala R., Arithmetic of linear algebraic groups over 2-dimensional geometric fields, Duke Math J. 121 (2004), pp 285 - 341. MR2034644 (2005f:11063)

[CTSk] Colliot-Thélène J.-L. and Skorobogatov J.-L., Groupe de Chow des zéro-cycles sur les fibrés en quadratiques, K-Theory $\mathbf{7}$ (1993), pp 477 - 500. MR1255062 (95c:14012)

[EL] Elman R. and Lam T.Y., Classification theorems for quadratic forms over fields, Comment. Math. Helv. 49(1974), pp 373 - 381. MR0351997 (50:4485)

[ELP] Elman R., Lam T.Y. and Prestel A., On some Hasse principles over formally real fields, Math. Z. 134(1973), pp 291 - 301. MR0330045 (48:8384)

[G] Gille P., La $R$-équivalence sur les groupes algébriques réductifs, Publications mathématiques de l'IHÉS. 86 (1997), pp 199 - 235. MR1608570 (99c:20066)

[Ga] Garibaldi S., Notes on $R G(F)=G(F)$ for $G$ adjoint of classical $D_{4}$ type, Unpublished, (2003).

[KMRT] Knus M.-A., Merkurjev A.S., Rost M. and Tignol J.-P., The Book of Involutions, AMS Colloquium Publication 44, 1998. MR1632779 (2000a:16031)

[KS] Kaito K. and Saito S., Unramified class field theory of arithmetical surfaces, Ann. of Math. 118(1983), pp 241 - 275. MR0717824 (86c:14006)

[L] Lam T.Y., Algebraic Theory of Quadratic Forms, W.A. Benjamin, 1973. MR0396410 (53:277)

[Ma] Manin Yu. I., Cubic forms, Amsterdam, North-Holland, 1974. MR0460349 (57:343)

[Me1] Merkurjev A.S., On the norm residue symbol of degree 2, Doklady Akad. Nauk SSSR 261(1981), pp 542 - 547, English translation: Soviet Math. Dokl. 24(1981), pp $546-$ 551. MR0638926 (83h:12015)

[Me2] Merkurjev A.S., $R$-equivalence and rationality problem for semisimple adjoint classical algebraic groups, Publications mathématiques de l'IHÉS. 84 (1996), pp 189-213. MR1441008 (98d:14055)

[Me3] Merkurjev A.S., Rost invariants of simply connected algebraic groups (with a section by Skip Garibaldi), Cohomological invariants in Galois cohomology, Univ. Lecture Ser. 28, 101-158, Amer. Math. Soc., Providence, RI, 2003. MR1999385

[MPT] Merkurjev A.S., Parimala R. and Tignol J.-P., Invariants of quasi-trivial tori and the Rost invariant, St. Petersburg Math. J. 14 (2003), pp 791 - 821. MR1970336 (2004c:11045) 
[MT] Merkurjev A.S. and Tignol J.-P., The multipliers of similitudes and the Brauer group of homogeneous varieties, J. reine angew. Math. 461 (1995), pp 13-47. MR1324207 (96c:20083)

[MH] Milnor J. and Husemoller D., Symmetric bilinear forms, Springer-Verlag, 1973. MR0506372 (58:22129)

[PR] Platonov V. and Rapinchuk A., Algebraic groups and number theory, Academic Press Inc., 1994. MR1278263 (95b:11039)

[P] Prestel A., Quadratische Semi-Ordnungen und quadratische Formen, Math. Z. 133(1973), pp 319 - 342. MR0337913 (49:2682)

[S] Sansuc J.-J., Groupe de Brauer et arithmétique des groupes algébriquessur un corps de nombres, J. reine angew. Math. 327 (1984), pp 13-81. MR0631309 (83d:12010)

[Sc] Scharlau W., Quadratic and Hermitian forms, Springer-Verlag, 1985. MR0770063 (86k:11022)

[Se] Serre J-P., Galois Cohomology, Springer-Verlag, 1997. MR1466966 (98g:12007)

[T] Tits J., Classification of algebraic semisimple groups, Algebraic groups and discontinuous subgroups (edited by Borel and Mastow), pp 33-62, American Mathematical Society, 1966. MR0224710 (37:309)

[V] Voskresenskiǔ V.E., Algebraic groups and their birational invariants, Translations of Mathematical Monographs 179, American Mathematical Society, 1998. MR1634406 (99g:20090)

[VK] Voskresenskiǔ V.E. and Klyachko A.A., Toroidal Fano varieties and root system, Math. USSR Izvestiya 24(1985), pp 221 - 244. MR0740791 (85k:14024)

[W] Wadsworth A.R., Merkurjev's elementary proof of Merkurjev's theorem, Applications of algebraic K-Theory to algebraic geometry and number theory, Contemp. Math, Vol 55.2, American Mathematical Society, 1986, pp 741 - 776. MR0862663 (88b:11078)

[We] Weil A., Algebras with involutions and the classical groups, J. Indian Math. Soc. (N.S.) 24(1960), pp 589 - 623. MR0136682 (25:147)

[Y] Yanchevskiı V.I., Whitehead groups and groups of $R$-equivalence classes of linear algebraic groups of non-commutative classical type over some virtual fields. Algebraic groups and arithmetic, Tata Inst. Fund. Res. Mumbai, 2004, pp 491-505. MR2094122 (2005h:20106)

School of Mathematics, Tata Institute of Fundamental Research, Homi Bhabha RoAd, Mumbai, India 400005

E-mail address: amitk@math.tifr.res.in

School of Mathematics, Tata Institute of Fundamental Research, Homi Bhabha RoAd, Mumbai, India 400005

Current address: Department of Mathematics and Computer Science, Emory University, Atlanta, Georgia 30322

E-mail address: parimala@mathcs.emory.edu 